\title{
Rotation and magnetic activity of the Hertzsprung-gap giant 31 Comae $^{\star, \star \star}$
}

\author{
K. G. Strassmeier ${ }^{1}$, T. Granzer ${ }^{1}$, M. Kopf ${ }^{1}$, M. Weber ${ }^{1}$, M. Küker ${ }^{1}$, P. Reegen ${ }^{2}$, J. B. Rice ${ }^{3}$, J. M. Matthews ${ }^{4}$, \\ R. Kuschnig ${ }^{2}$, J. F. Rowe ${ }^{5}$, D. B. Guenther ${ }^{6}$, A. F. J. Moffat ${ }^{7}$, S. M. Rucinski ${ }^{8}$, D. Sasselov ${ }^{9}$, and W. W. Weiss ${ }^{2}$ \\ 1 Astrophysical Institute Potsdam (AIP), An der Sternwarte 16, 14482 Potsdam, Germany \\ e-mail: kstrassmeier@aip.de \\ 2 Institut für Astronomie, Universität Wien, Türkenschanzstraße 17, 1180 Wien, Austria \\ e-mail: reegen@astro.univie.ac.at \\ 3 Department of Physics, Brandon University, Brandon, Manitoba R7A 6A9, Canada \\ e-mail: rice@BrandonU.ca \\ 4 Department of Physics and Astronomy, University of British Columbia, 6224 Agricultural Rd., Vancouver, British Columbia, \\ Canada, V6T $1 Z 1$ \\ 5 NASA Ames Research Center, Moffett Field, CA 94035, USA \\ 6 Department of Astronomy and Physics, St. Mary's University Halifax, NS B3H 3C3, Canada \\ 7 Département de Physique, Université de Montréal C.P. 6128, Succ. Centre-Ville, Montréal, QC H3C 3J7, Canada \\ 8 Department of Astronomy \& Astrophysics, University of Toronto, 50 St. George Str., Toronto, OM5S 3H4, Canada \\ 9 Harvard-Smithsonian Center for Astrophysics 60 Garden Street, Cambridge, MA 02138, USA
}

Received 20 May 2010 / Accepted 15 July 2010

\section{ABSTRACT}

\begin{abstract}
Context. The single rapidly-rotating G0 giant 31 Comae has been a puzzle because of the absence of photometric variability despite its strong chromospheric and coronal emissions. As a Hertzsprung-gap giant, it is expected to be at the stage of rearranging its moment of inertia, hence likely also its dynamo action, which could possibly be linked with its missing photospheric activity.

Aims. Our aim is to detect photospheric activity, obtain the rotation period, and use it for a first Doppler image of the star's surface. Its morphology could be related to the evolutionary status.

Methods. We carried out high-precision, white-light photometry with the MOST satellite, ground-based Strömgren photometry with automated telescopes, and high-resolution optical echelle spectroscopy with the new STELLA robotic facility.

Results. The MOST data reveal, for the first time, light variations with a full amplitude of 5 mmag and an average photometric period of $6.80 \pm 0.06$ days. Radial-velocity variations with a full amplitude of $270 \mathrm{~m} \mathrm{~s}^{-1}$ and a period of $6.76 \pm 0.02$ days were detected from our STELLA spectra, which we also interpret as due to stellar rotation. The two-year constancy of the average radial velocity of $+0.10 \pm 0.33 \mathrm{~km} \mathrm{~s}^{-1}$ confirms the star's single status, as well as the membership in the cluster Melotte 111. A spectrum synthesis gives $T_{\text {eff }}=5660 \pm 42 \mathrm{~K}, \log g=3.51 \pm 0.09$, and $[\mathrm{Fe} / \mathrm{H}]=-0.15 \pm 0.03$, which together with the revised Hipparcos distance, suggests a mass of $2.6 \pm 0.1 M_{\odot}$ and an age of $\approx 540 \mathrm{Myr}$. The surface lithium abundance is measured to be nearly primordial. A detection of a strong He I absorption line indicates nonradiative heating processes in the atmosphere. Our Doppler images show a large, asymmetric polar spot, cooler than $T_{\text {eff }}$ by $\approx 1600 \mathrm{~K}$, and several small low-to-mid latitude features that are warmer by $\approx 300-400 \mathrm{~K}$ and are possibly of chromospheric origin. We computed the convective turnover time for $31 \mathrm{Com}$ as a function of depth and found on average $\tau_{\mathrm{C}} \approx 5$ days.

Conclusions. 31 Com appears to be just at the onset of rapid magnetic braking and Li dilution because its age almost exactly coincides with the predicted onset of envelope convection. That we recover a big polar starspot despite the Rossby number being larger than unity, and thus no efficient (envelope) dynamo is expected, leads us to conclude that 31 Com still harbors a fossil predominantly poloidal magnetic field. However, the increasing convective envelope may have just started an interface dynamo that now is the source of the warm surface features and the corresponding UV and X-ray emission.
\end{abstract}

Key words. stars: activity - stars: atmospheres - stars: individual: 31 Comae - starspots - stars: late-type - stars: rotation

* Based on data obtained with the MOST satellite, a Canadian Space Agency mission, operated jointly by Dynacon, Inc., and the Universities of Toronto and British Columbia, with assistance from the University of Vienna; the STELLA robotic telescope in Tenerife, an AIP facility jointly operated by AIP and IAC, and the Vienna Automatic Photoelectric Telescopes in Arizona, jointly operated by the University of Vienna and AIP.

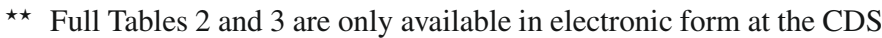
via anonymous ftp to

cdsarc.u-strasbg.fr $(130.79 .128 .5)$ or via

http://cdsarc.u-strasbg.fr/viz-bin/qcat?J/A+A/520/A52

\section{Introduction}

The G0III star 31 Com=HD 111812 is one of the few single giants that exhibit very rapid rotation despite not being a member of a close-binary system and thus appear magnetically overactive. A Ca II H \& K emission-line flux of nearly $10^{7} \mathrm{erg} \mathrm{cm}^{-2} \mathrm{~s}^{-1}$ (Strassmeier et al. 1990), a $v \sin i$ of $60-70 \mathrm{~km} \mathrm{~s}^{-1}$ (e.g. Reiners 2006), and its apparent single status and giant luminosity (Bopp $\&$ Stencel 1981; Gray et al. 2001a) indicates a relation with the G7III single-star FK Com, the prominent prototype of the FKComae class of stars (e.g. Korhonen et al. 2001, 2009). However, the evolutionary status of these two objects is rather different, 
with the latter thought to be the merger of a previously contact binary.

Early IUE observations of 31 Com by Simon (1984) showed the presence of highly excited chromospheric and transitionregion emission lines. The star is also a strong X-ray emission source detected during the ROSAT all-sky survey (Hünsch et al. 1998; for a summary of other measures see Gondoin 1999, 2005), but it exhibits a deficient X-ray luminosity compared to the coronal proxy $\mathrm{C}$ IV and with respect to mean trends defined by G-K dwarfs (Simon \& Drake 1989). Its coronal properties have been studied more recently with XMM-Newton by Scelsi et al. (2004), who find that the star's coronal magnetic loops must peak at a temperature near $10^{7} \mathrm{~K}$. Ayres et al. (1998, 2007) find that the coronal and transition-region lines are superrotationally broadened, e.g., the Fe XXI profile of 31 Com appears with an $F W H M$ of $\approx 280 \mathrm{~km} \mathrm{~s}^{-1}$, more than twice as wide as expected from $v \sin i$. A correlation between excess line broadening and $v \sin i$ suggests that transition-zone gas extends out to about one stellar radius. Ayres et al. (1998) were the first to postulate that the X-ray deficiency, the lack of photometric variability, and the apparent highly extended transition region could be understood if the stellar corona was in fact seated in a relic dipole magnetosphere left over from the main sequence phase of the star. The large line broadening, on the other hand, could be explained by the large gas extension and by transient explosive activity as we know it from the Sun (see Ayres et al. 2007).

On the contrary, numerous attempts to determine a rotation period based on photometric variations failed. Strassmeier \& Hall (1988) concluded that 31 Com does not vary in light but noted that the scatter in their $V$ data was about twice as high during the 1983-84 season as in the middle of the following 1984-85 season. Their external uncertainties of the combined data never exceeded \pm 0.009 mag in $V .31$ Com is clearly not an easy target to detect rotational modulation from groundbased data. An IUE search for rotational modulation of ultraviolet emission-line fluxes produced negative results as well (Simon 1986). Even our long-term APT observations did not lead to a conclusive period (Strassmeier et al. 1999) although signals at 6.96 days and 2.31 days were present in the $U$-band APT data but were formally below the limit of significance, i.e. had an amplitude signal-to-noise ratio of below $4: 1$ according to the criteria by Breger et al. (1993). However, the star seemed to show seasonal brightness changes in all bandpasses that were already noted in earlier observations by Lockwood et al. (1993). The Lowell team obtained an rms error from eight years of Strömgren data of as small as 0.0029 , i.e. a pretty constant star for ground-based photometry, but mentioned that $31 \mathrm{Com}$ is variable on a seasonal timescale. Strassmeier et al. (1997a) presented a plot of seasonal photometry over almost 13 years that also showed the long-term constancy of $31 \mathrm{Com}$. Nevertheless, this led Kazarovets et al. (2003) to include 31 Comae in the 77th name list of variable stars as LS Comae.

31 Comae is most interesting because of its evolutionary status as a Hertzsprung-gap star and a possible member of the close Coma-Berenices cluster Melotte 111 (Bounatiro 1993; Odenkirchen et al. 1998). The vast majority of early-G stars are comparably slow rotators, but some have rotational line broadening up to $100 \mathrm{~km} \mathrm{~s}^{-1}$ (Hagen \& Stencel 1985; Gray 1989). The sudden decline in rotation at spectral type G0-3III in the sample investigated by Gray (1989) partly results from a mix of stars of different masses and ages associated with the rapid evolution into the Hertzsprung gap (De Medeiros \& Mayor 1999; De Medeiros et al. 2000) and partly from deep mixing (Böhm-Vitense 2004). As a G0III star, 31 Com is in a pre-braking phase but already started a rearrangement of its internal moment of inertia as the hydrogen-burning shell expands rather quickly at that stage. For a $2.5-M_{\odot}$ star this phase lasts for $\approx 6$ Myr according to the models with overshooting from Pietrinferni et al. (2004) and therefore Hertzsprung-gap stars are rather rare. Bright "sister stars" of 31 Com are known though, e.g. HR 9024 (G1III), $\psi^{3}$ Psc (G0III), and $35 \mathrm{Cnc}=\mathrm{HR} 3387$ (G0III) (Wallerstein et al. 1994; Bopp et al. 1988), but also the G0-1III primary of the Capella binary system (Ayres \& Linsky 1980) resembles 31 Com.

In this paper, we first present and describe our new MOST data and a set of new photometric APT observations along with earlier data (Sect. 2). In the same section, we also present new long-term high-resolution spectroscopy of 31 Com obtained at STELLA, KPNO, and CFHT. In Sect. 3, we apply various period-search algorithms to the photometry and the radial velocities. The MOST observations revealed, for the first time, the flux modulation due to the rotation of the star. In Sect. 3.4 we analyze the STELLA radial velocities in the same way as the photometry. A more in-depth determination and a discussion of 31 Comae's stellar parameters are presented in Sect. 4. Section 5 presents the first attempt to Doppler image the surface of 31 Comae followed by our discussion (Sect. 6) and conclusions (Sect. 7).

\section{Acquisition of photometric and spectroscopic data}

Table 1 is a summary of all observations in this paper. In particular, it lists the total number of individual spectra and light-curve points, $N$, for the individual telescopes, respectively. Telescopes are indicated by their short name, CF is the $0.9 \mathrm{~m}$ coude feed telescope at Kitt Peak, and STELLA is the AIP robotic facility in Tenerife. PMT stands for photomultiplier tube.

\subsection{MOST photometry from 2007}

MOST observed 31 Com $\left(V=4^{\mathrm{m}} \cdot 9, B-V=0.67\right)$ from March 15 to 25,2007 . In that period the star was acquired during about half of each $101 \mathrm{~min}$ spacecraft orbit, the remaining time was used to observe another field. The data were obtained in the Direct Imaging field producing a PSF with $\approx 2.5$ pixel $F W H M\left(3\right.$ arcsec pixel $\left.^{-1}\right)$ rendered in $20 \times 20$ pixel subrasters of the $1 \mathrm{k} \times 1 \mathrm{k} \mathrm{CCD}$ frame. With an exposure time of $0.3 \mathrm{~s}$ plus accounting for frame transfer and readout time an image was gathered every $0.8 \mathrm{~s}$. To conform with data transfer limitations, 52 images were stacked together onboard the satellite. Data extraction was performed on stacked images obtained every $42 \mathrm{~s}$ (effective sampling time). The constant A-star HD 112152 $(V=9 \mathrm{~m} .05, \mathrm{~A} 3 \mathrm{~V})$ was observed during the same time interval and with the same sampling as $31 \mathrm{Com}$ and is shown in Fig. 1a for comparison. Its data were additionally binned in units of the MOST orbital period (101 $\mathrm{min})$ to increase the signal. The data reduction scheme (Rowe et al. 2006) is based on conventional aperture photometry with an additional correction for straylight contribution. The $31 \mathrm{Com}$ photometry reveals a point-to-point scatter of 0.9 mmag. The corresponding light curve is shown in Fig. 1a.

\subsection{APT Strömgren and Johnson photometry from 1997-2003}

Continuous photometry was obtained with the two $0.75 \mathrm{~m}$ T6 \& T7 Vienna University Automatic Photoelectric Telescopes 
Table 1. Summary of new observations in this paper.

\begin{tabular}{lllll}
\hline \hline Observatory & Type & Instr. & $\begin{array}{l}\text { Time range } \\
\text { JD 24+ }\end{array}$ & $N$ \\
\hline \hline CFHT & Coudé spec. & Gecko & 49344 & 4 \\
KPNO CF & Coudé spec. & Coudé & $49415-50925$ & 93 \\
APT T6 & $v y$ & PMT & $50117-50261$ & 380 \\
APT T1 & $U B V$ & PMT & $50768-52820$ & 458 \\
APT T7 & $V I_{\mathrm{C}}$ & PMT & $50855-50929$ & 143 \\
APT T6 & $b v$ & PMT & $52390-52406$ & 239 \\
MOST & Optical phot. & CCD & $54174-54184$ & 11679 \\
STELLA-I & Echelle spec. & SES & $54175-54527$ & 187 \\
\hline
\end{tabular}

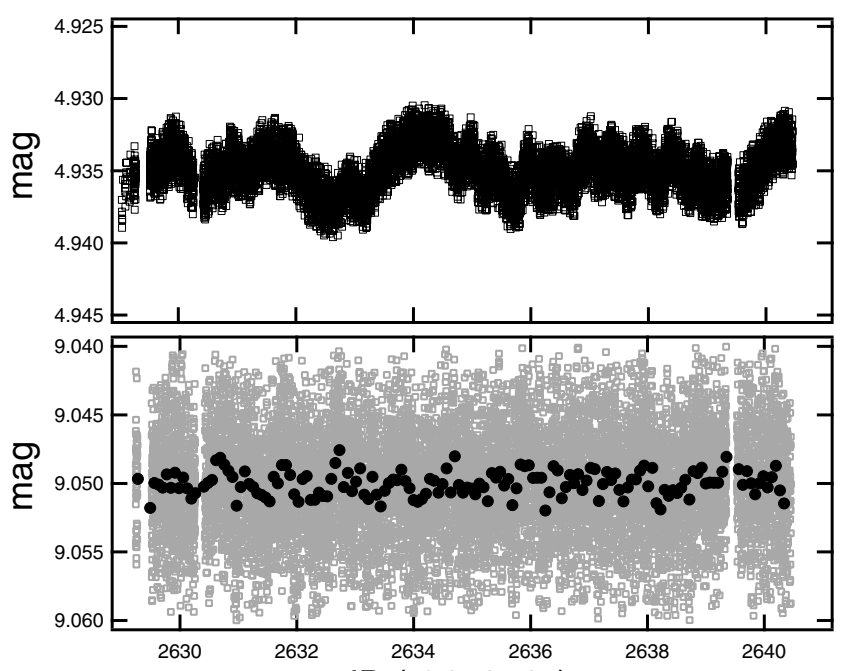

a)

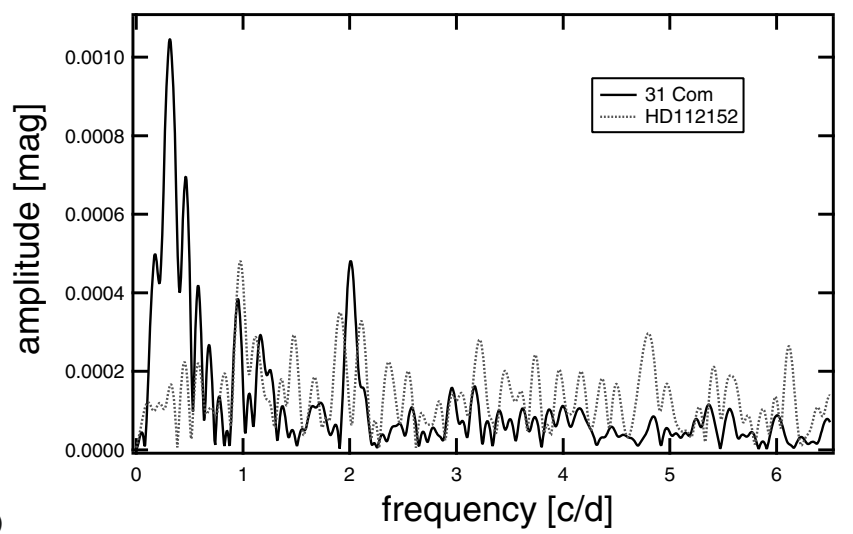

Fig. 1. MOST photometry of $31 \mathrm{Com}$ in March 2007. a) Light curve for a continuous 11-day run (top panel) and of a fainter comparison star (lower panel; HD 112152, A3V; unbinned data are shown as grey-scale dots, binned data as black dots). The abscissa is in fractional days from JD 2451545 and the magnitudes are MOST instrumental magnitudes. b) DFT periodogram from the MOST data. The full line is $31 \mathrm{Com}$, the dashed line is for the comparison star HD 112152. The abscissa is in units of cycles per day.

(APT) and the $0.25 \mathrm{~m}$ T1 Phoenix telescope, both located at Fairborn Observatory in Arizona (Strassmeier et al. 1997b, 2001). The T6 data are the result of a contemporaneous run from February 4 to June 27, 1996 (76 nights). The number of data points is 378 in Strömgren $b$ and 385 in $y$. The $v b$ data are the result of a continuous 16-night campaign with T6 in May 2002 and consist of 239 points in Strömgren $v$ and 232 points in $y$ with 30 readings per night on average. Each one of these is actually a mean of three individual interpolations between the variable and the comparison star (see Granzer et al. 2001).

The T1 $U B V$ and $\mathrm{T} 7 V I_{\mathrm{C}}$ data are a continuation of the 1996/97 data already described and analyzed in Strassmeier et al. (1997a,1999) and were taken on a single-reading-per-night basis with T1 from fall 1997 until spring 2003 and with T7 until 1998. Table 1 summarizes the exact time ranges of all new observations in this paper. We removed 31 Com from the target list of T7 but kept it on T1 until its retirement in late 2003 in order to monitor 31 Com's long-term brightness. These data are presented in their original form in the electronic attachment but are not used in the present analysis due to their moderate $1 \%$-like precision.

All APT observations were made differentially with respect to HD 111469 as the comparison star $(V=5$. $78, B-V=0.03$; Nicolet 1978) and HD $113996(V=4.80, B-V=1.48$; Moffett \& Barnes 1979) as the check star. All three telescopes reached approximately one million counts for integrations of $10 \mathrm{~s}$ (30 s in $U$ for T1), and thereby achieved an external precision of 1.2 mmag (T6 in $y$ ), 4 mmag (T7 in $V$ ), and $10 \mathrm{mmag}$ (T1 in $V$ ).

\subsection{STELLA echelle spectroscopy from 2007-2008}

From Mar 16, 2007 to Mar 22, 2008 we obtained 187 highresolution echelle spectra with the $1.2 \mathrm{~m}$ robotic STELLA-I telescope and its fiber-fed STELLA Echelle Spectrograph (SES; Strassmeier et al. 2004). During the period Mar 10-22, 2008 the one-picks-per-night observing schedule was changed to five picks per night to search for short-term variations. All spectra cover the wavelength range from $390-880 \mathrm{~nm}$ with increasing inter-order gaps starting at $720 \mathrm{~nm}$. Together with its $2 \mathrm{k} \times 2 \mathrm{k}$, $13.5-\mu \mathrm{m}$ E2V CCD it provides an effective resolving power of $R=55000$ corresponding to a spectral resolution of $5.5 \mathrm{~km} \mathrm{~s}^{-1}$ at $650 \mathrm{~nm}$. Exposure time was set to $800 \mathrm{~s}$ and yielded $S / N$ ratios between 300-100:1 (per resolution bin) depending upon weather conditions. Reference spectra of MK-standard stars as well as radial-velocity standards were obtained throughout the entire observing period.

The echelle spectra were automatically reduced and extracted using the IRAF-based STELLA data-reduction pipeline (see Weber et al. 2008). The images were corrected for bad pixels and cosmic rays. Bias levels were removed by subtracting the average overscan from each image followed by the subtraction of the mean of the (already overscan subtracted) master bias frame. The target spectra were flattened through a division by the master flat which had been normalized to unity. Radial velocities from STELLA-SES spectra were derived from an orderby-order cross correlation with a synthetic template spectrum and then averaged. The standard error of a STELLA-SES observation of $31 \mathrm{Com}$ is $\approx 100 \mathrm{~m} \mathrm{~s}^{-1}$. Individual observations are given in Table 2. No systemic zero point shift was added. The rms value given is the rms from cross correlations of 19 spectral orders. The complete data set is available in electronic form at the CDS.

\subsection{KPNO coudé spectroscopy from 1994-1998}

Altogether 93 spectra were obtained at Kitt Peak National Observatory (KPNO) with the 0.9 m coudé feed telescope during runs in Mar. 4-18, 1994, Feb. 24-Mar. 7, 1995, Jan. 11-26, 1996, Dec. 26-Jan. 15, 1997/98, and April 3-22, 1998. In 1994-1996, a $800 \times 800$ TI CCD (TI-5 chip, $15 \mu$ pixels) 
Table 2. Barycentric STELLA radial velocities.

\begin{tabular}{rlll}
\hline \hline UT Date & $\begin{array}{l}\text { HJD } \\
(2400000+)\end{array}$ & $\begin{array}{l}v_{\mathrm{r}} \\
\left(\mathrm{km} \mathrm{s}^{-1}\right)\end{array}$ & $\begin{array}{l}\mathrm{rms} \\
\left(\mathrm{km} \mathrm{s}^{-1}\right)\end{array}$ \\
\hline 2008 Mar 22 & 54547.757805 & 0.215 & 0.087 \\
22 & 54547.697025 & 0.243 & 0.088 \\
22 & 54547.628012 & 0.068 & 0.081 \\
22 & 54547.523352 & 0.134 & 0.084 \\
21 & 54547.416795 & 0.764 & 0.103 \\
19 & 54544.705020 & 0.059 & 0.096 \\
19 & 54544.632086 & 0.470 & 0.092 \\
19 & 54544.561231 & 0.769 & 0.105 \\
19 & 54544.541172 & 1.076 & 0.096 \\
\hline
\end{tabular}

Notes. Full Table 2 is available at the CDS.

Table 3. KPNO radial velocities.

\begin{tabular}{rllll}
\hline \hline UT Date & $\begin{array}{l}\text { HJD } \\
(2400000+)\end{array}$ & $\begin{array}{l}v_{\mathrm{r}} \\
\left(\mathrm{km} \mathrm{s}^{-1}\right)\end{array}$ & $\begin{array}{l}\mathrm{rms} \\
\left(\mathrm{km} \mathrm{s}^{-1}\right)\end{array}$ & $\begin{array}{l}\text { Ref. } \\
\text { star }\end{array}$ \\
\hline 1994 Mar 04 & 49415.8495 & +1.6 & 4.4 & $16 \mathrm{Vir}$ \\
04 & 49415.8612 & +1.3 & 4.2 & $16 \mathrm{Vir}$ \\
05 & 49416.7463 & +1.3 & 3.8 & $\delta$ Aur \\
05 & 49416.7532 & +1.0 & 4.9 & $\delta$ Aur \\
06 & 49417.7498 & -1.9 & 4.9 & $16 \mathrm{Vir}$ \\
07 & 49418.7465 & -2.3 & 4.3 & $16 \mathrm{Vir}$ \\
10 & 49421.7481 & -2.6 & 4.5 & $16 \mathrm{Vir}$ \\
11 & 49422.7509 & -2.4 & 4.2 & $16 \mathrm{Vir}$ \\
12 & 49423.9013 & -2.5 & 3.9 & $16 \mathrm{Vir}$ \\
\hline
\end{tabular}

Notes. Full Table 3 is available at the CDS.

was used with grating A, camera 5 and the long collimator at $R=38000$ that gave a $8-\mathrm{nm}$ wavelength range centered at $642 \mathrm{~nm}$. An exposure time of $1200 \mathrm{~s}$ resulted on average in a $S / N$ of 200:1. From 1998 on, we used the $3 \mathrm{k} \times 1 \mathrm{k}$ Ford3k CCD that gave a wavelength coverage of $30 \mathrm{~nm}$ but with a lower resolution of $R=27000$ at $650 \mathrm{~nm}$ but comparable $S / N$.

All data were reduced in the standard fashion using IRAF and included standard bias subtraction, flat fielding and aperture extraction. Frequent wavelength comparison spectra and spectra of bright radial-velocity standards were obtained several times throughout a night to ensure an accurate wavelength calibration.

Radial velocities from the KPNO spectra were derived from cross correlating the 31 Com spectra mostly with the IAU velocity standard 16 Vir (K0.5III, $\left.v_{\mathrm{r}}=+35.7 \mathrm{~km} \mathrm{~s}^{-1}\right)$. Occasionally, other IAU standards were employed, i.e. $\delta$ Aur $\left(\mathrm{K} 0 \mathrm{III}, v_{\mathrm{r}}=\right.$ $+7.61 \mathrm{~km} \mathrm{~s}^{-1}$ ), $\alpha$ Tau (K5III, +54.1 km s${ }^{-1}$ ), HR 3145 (K2III, $+70.90 \mathrm{~km} \mathrm{~s}^{-1}$ ), and $\beta$ Gem (KOIII, $+3.30 \mathrm{~km} \mathrm{~s}^{-1}$ ). The standard error of a KPNO observation is $4-7 \mathrm{~km} \mathrm{~s}^{-1}$. This is anomalously high for spectra at the given resolution but is due to the broad line profiles of $31 \mathrm{Com}$. Individual observations and the adopted cross-correlation reference star are given in Table 3. From 1994-1995 the error of a single unit-weight velocity was $4.5 \mathrm{~km} \mathrm{~s}^{-1}$. In 1996, a CCD with smaller pixels but also smaller wavelength coverage was used and the uncertainties were accordingly larger, $\approx 7 \mathrm{~km} \mathrm{~s}^{-1}$. In 1998, the larger wavelength coverage with a bigger CCD reduced this again to $4.0 \mathrm{~km} \mathrm{~s}^{-1}$. The cross-correlation reference stars 16 Vir, $\delta$ Aur, HR 3145, $\alpha$ Tau, and $\beta \mathrm{Gem}$ were used with an adopted velocity of $+35.70,+7.61$, $+70.90,+54.1$ and $+3.30 \mathrm{~km} \mathrm{~s}^{-1}$, respectively. The complete data set is available in electronic form at the CDS.

\subsection{CFHT coudé spectroscopy from 1993}

Strassmeier et al. (1994) presented a subset of the CFHT spectra obtained in December 1993. A total of four spectra, covering the wavelength range $633-653 \mathrm{~nm}$ and one covering the lithium $671 \mathrm{~nm}$ region, were obtained. We used the f/ 8 coudé spectrograph with the $6001 \mathrm{~mm}^{-1}$ mosaic grating in first order. The effective resolving power was 27000 with the $2 \mathrm{k} \times 2 \mathrm{k}$ Lick CCD and allowed a $S / N$ ratio of close to 400:1 in $900 \mathrm{~s}$.

\section{Rotational period search}

\subsection{Summary of techniques}

Four different techniques are applied in the course of this paper:

- Phase dispersion minimization (PDM, Lafler \& Kinman 1965; Stellingwerf 1978) is a reliable approach to nonsinusoidal variations such as rotational modulations caused by stellar surface structure. Its advantage over the Fourier methods (especially if not more than a single-periodic process is considered) is that no initial assumption on the shape of the signal needs to be drawn.

- SigSpec, developed by Reegen (2007), relies on a frequency- and phase-dependent statistical treatment of the discrete Fourier transform (DFT). For a given DFT amplitude $A$, it provides unbiased spectral significance $\operatorname{sig}(A)$, directly linked to false-alarm probabilities $\Phi_{\mathrm{FA}}(A)$ via

$\operatorname{sig}(A)=-\log \Phi_{\mathrm{FA}}(A)$,

taking into account the properties of the time-domain sampling appropriately.

- The Lomb-Scargle (L-S) periodogram is designed for unevenly spaced data. Lomb (1976) proposed to use leastsquares fits to sinusoidal curves and Scargle (1982) extended this by deriving the null distribution for it. We use the formulation implemented by Press et al. (2002).

- CLEAN was originally invented to clean radio maps from noise (Roberts et al. 1987). Applied to time series it removes iteratively the artificial frequencies introduced due to the window function.

\subsection{Application to MOST photometry}

Figure 1a shows the MOST photometry and Fig. 1b a DFT periodogram over a large period range. It reveals a clear peak at $\approx 0.31 \mathrm{c} / \mathrm{d}$, or $\approx 3.2$ days. Its window function is sufficiently clean, and our expected rotational signal is not affected by the fact that 31 Com was not observed exclusively during each spacecraft orbit of $101.4 \mathrm{~min}(14.2 \mathrm{c} / \mathrm{d}$; Walker et al. 2003). The $\approx 0.3 \mathrm{c} / \mathrm{d}$ peak is confirmed by the L-S approach (Fig. 2a) which provides a refined period of $1 \pm 2$ days.

The phase-dispersion diagram in Fig. $2 \mathrm{~b}$ reveals two pronounced local minima at periods of 3.4 and 6.8 days. While the 3.4-day minimum is well-defined and symmetric, the 6.8day minimum appears split into two equal minima at 6.88 and 6.45 days. In addition, there is a trend towards a lower phase dispersion with increasing period. This addresses a general PDM-related problem for periods reaching the order of the time spanned by the data set. In this case, the number of phase bins containing data from different periods becomes poor, and even for periods not referring to an intrinsic signal, the variance within the bins will remain of the order of the total variance because the high systematic deviations of data originating from different cycles are missing. If we remove this trend to first order, 
a)

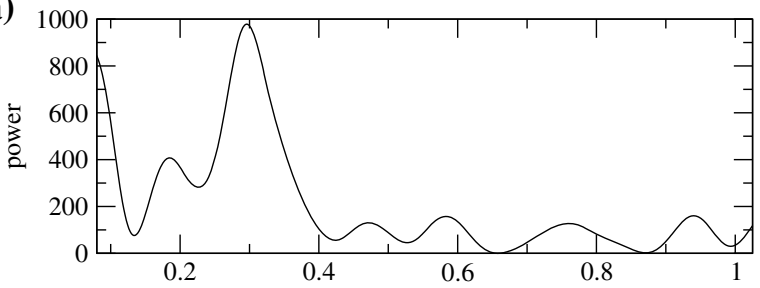

b)

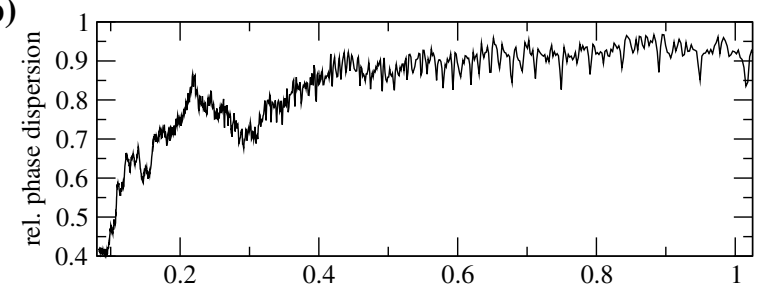

c)

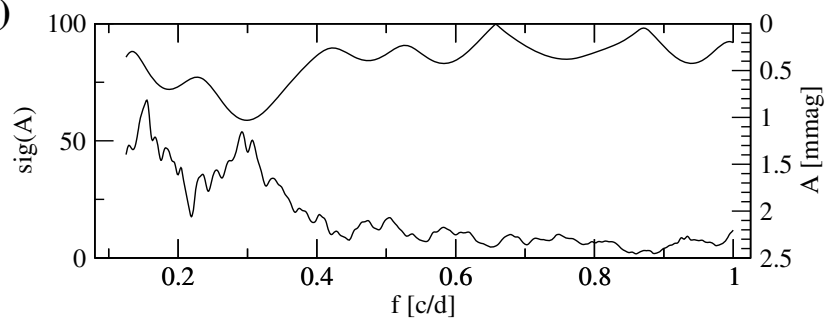

Fig. 2. Periodograms for the MOST data with a) the Lomb-Scargle algorithm, b) the Phase-Dispersion Minimization (PDM), and c) a statistical treatment of the Discrete Fourier Transform (DFT) as formulated by Reegen (2007). The DFT amplitude, $A$, in mmag (top curve), is shown on the right ordinate (inverted) and its significance, sig $(A)$, on the left ordinate. A peak of $\approx 0.3 \mathrm{c} / \mathrm{d}$ is evident from all techniques.

i.e. by dividing the relative phase dispersion by a low-order polynomial fit to its upper envelope, the 3.4-day minimum appears $36 \%$ deeper than the 6.8-day minimum.

The DFT application with SIGSPEC uses a fundamental frequency $f$ plus a set of harmonics, i.e. $f_{\mathrm{h}}=(h+1) f, h=$ $0,1, \ldots, H$. As discussed in detail by Reegen et al. (2006), MOST light curves potentially suffer from stray-light induced artifacts, which produce pseudo-periodicities close to the orbital period of the spacecraft (101.4 min). Since the rotation period of the star is expected to be considerably longer than 1 day, setting $H=12$ avoids a contamination of the frequencies below $1 \mathrm{~d}^{-1}$ by the stray-light artifacts around $14.2 \mathrm{~d}^{-1}$.

A combined DFT amplitude and significance spectrum is displayed in Fig. 2c. The greatest significance is associated with an amplitude peak of $1.0 \mathrm{mmag}$ found at $0.143 \mathrm{c} / \mathrm{d}$. Comparing this frequency to the Rayleigh frequency resolution (the inverse length of the dataset) of $0.09145 \mathrm{c} / \mathrm{d}$, this peak has to be considered as an indication of a signal at a period too long to be resolvable by the DFT. However, the significance attains another maximum at $0.296 \mathrm{c} / \mathrm{d}$ which corresponds to a period of 3.378 days, in good agreement with the period found by PDM, Lomb-Scargle, and a standard FFT. Unfortunately, the high-amplitude peak at $0.143 \mathrm{c} / \mathrm{d}$ does not permit us to state whether twice the 3.378day period is also obtainable by DFT.

The advantage of PDM compared to DFT is that PDM does not imply any assumption on the shape of the examined signal, whereas the DFT implicitly relies on the sinusoids. If a signal is non-sinusoidal (as in case of the MOST data), the DFT spreads the power contained in this signal over the signal frequency and all integer multiples. A weakness of the PDM analysis is that each of the aforementioned local minima is superposed by several sharp peaks spaced by approximately the $101.4 \mathrm{~min}$ period of the MOST orbit. PDM recovers the 6.8-d period as the most significant.

In principle, very small photometric amplitudes or even non detections of rotational modulation of otherwise magnetically active stars may be due to several reasons. Firstly, a low inclination of the axis of rotation with respect to the observer may prevent the detection of rotational-modulation in the diskintegrated light curve. Secondly, a star's surface may show a large number of small faculae and/or spots distributed symmetrically over the surface at the same time (like a chessboard structure). In this case, simple local flux cancelation would prevent the detection of light changes by means of disk-integrated photometry. A third reason for a non detection could be that the relatively thin convective envelope of a G0 star may prohibit the formation of large-scale photospheric and chromospheric surface structure and result in a more uniform spot distribution. Because the MOST light curve shape appeared not perfectly repeated in the second stellar rotation, we conclude that it is most likely that the white-light bandpass of MOST is dominated by faculae rather than spots with a variability time scale significantly shorter than the rotational period. This is more of a solar analogy than what we would expect for a giant star rotating 30 times faster than the Sun.

\subsection{Application to APT photometry}

Each of the seasonal data sets was analyzed with the four algorithms described in Sect. 3.1. As we have seen from the application to the MOST data, not all algorithms provide a reliable recovery. Whenever possible, seasonal data were first split into subsets from the three telescope - T1, T6, and T7 - and analyzed individually. This appeared necessary because the zero-point shifts between the three telescopes (cf. Strassmeier et al. 1997b) likely exceed the expected amplitude of $31 \mathrm{Com}$ and would confuse or even suppress the rotational-modulation signal. The corresponding seasonal and instrumental amplitude spectra and spectral windows are not given in detail but all spectral windows show strong 1-day aliasing and the expected high degree of symmetry with respect to $0.5 \mathrm{c} / \mathrm{d}$. None of the T1 and T7 data showed a clear period above its respective noise levels and were not further investigated.

Only the 1996 and 2002 T6 data seem to have sufficient photometric precision that would allow the detection of an amplitude and period comparable to the MOST data. Because periods shorter than one day would lead to unrealistically large equatorial rotational velocities for a G0 giant, we considered only the range between 0 and $1 \mathrm{c} / \mathrm{d}$, claiming all frequencies exceeding this upper limit to be aliases of the "true" frequency. The frequency and amplitude of the highest peak in each amplitude spectrum are taken as initial values for a least-squares fit. Amplitude and period rms errors are computed by means of numerical simulations of error propagation as provided by the program EPSim (Reegen 2004).

As a cross check, we apply exactly the same SIGSPEC DFT analysis as for the MOST data and plot the significance spectra in Fig. 3. Again, the significance spectra represent the (negative) logarithm of the false alarm probability of the considered frequency plus 12 integer multiples. The rotational frequencies obtained from all four data sets are in acceptable agreement with the value obtained from the MOST photometry. A summary of all period determinations is provided in Table 4 . We adopt an average from the MOST photometry of $6.80 \pm 0.06$ by assuming equal weights for the different methods where the error is now simply its rms. Figures $4 \mathrm{a}, \mathrm{b}$ show phase plots for the MOST and 


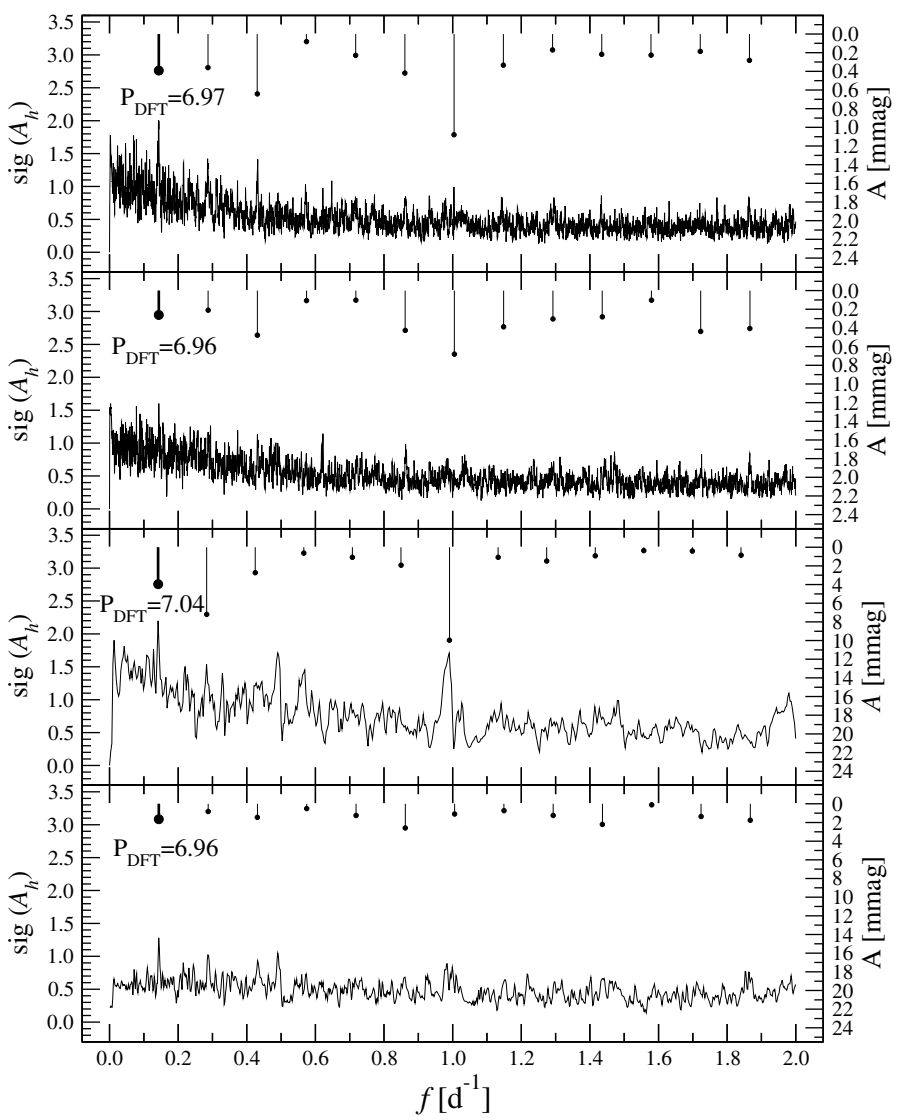

Fig. 3. Significance spectra of the APT light curves. From top to bottom: $b$ 1996, $y$ 1996, $v 2002, y 2002$. Note that the amplitude scale for 1996 and 2002 are different. For each frequency, $f$, the significance of this frequency and 12 integer multiples are added up, returning the FalseAlarm Probability for the fundamental and harmonics simultaneously, thus taking into account the expected non-sinusoidal photometric variation invoked by the rotating surface. The length of the pins referred to the right (inverted) ordinate and represent the best-fitting frequencies (Eq. (1)) and the individual amplitudes of all 12 harmonics.

Table 4. Best-fit periods in days from the MOST observations, the four APT T6 data sets, and the STELLA radial velocities.

\begin{tabular}{llllll}
\hline \hline Dataset & CLEAN & L-S & PDM & DFT & $\delta f_{\mathrm{R}}$ \\
\hline MOST 2007 & $\ldots$ & 3.377 & 6.87 & 6.97 & 0.091 \\
APT $b$ 1996 & 3.54 & 7.16 & 7.04 & 6.97 & 0.007 \\
APT $y$ 1996 & 3.56 & 3.56 & 6.94 & 6.96 & 0.007 \\
APT $v$ 2002 & 8.31 & $\ldots$ & 3.47 & 7.04 & 0.062 \\
APT $y$ 2002 & 12.2 & $\ldots$ & 3.45 & 6.96 & 0.061 \\
STELLA 2007/8 & 6.73 & 6.76 & 6.80 & 6.75 & 0.008 \\
\hline
\end{tabular}

Notes. The methods applied are CLEAN, L-S=Lomb Scargle, $\mathrm{PDM}=$ Phase Dispersion Method, DFT=Discrete Fourier Transform. The last column lists the Rayleigh frequency resolution, $\delta f_{\mathrm{R}}$, defined as the inverse total time covered by the respective time series.

the APT photometry, respectively. The MOST data are additionally plotted with half of the adopted period for visual comparison.

\subsection{Application to STELLA radial velocities}

\subsubsection{Mean radial velocities and error estimates}

We measured a total of 187 radial velocities with STELLA between 2007-2008, 93 radial velocities at KPNO between a) MOST lightcurve
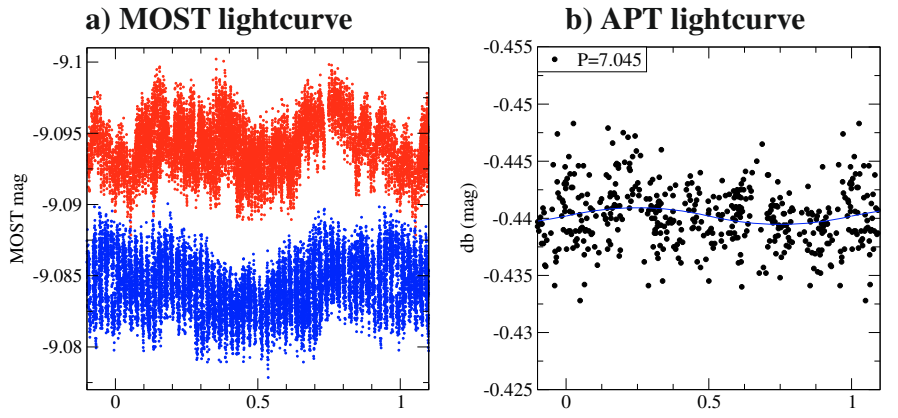

Fig. 4. Phased light curves. a) The MOST light curve from 2007. Shown are the light curves phased with the 6.80-day (bottom) and the 3.38-day period (top). b) Our best-sampled APT light curve with a period of 7.045 days (data set APT $b 1996$ in Table 4). The zero phase is arbitrary.

1994-1998, and five at CFHT in 1993. Not surprisingly, all these measures have different zero points. Moreover, the KPNO data were obtained with different setups during a total of five observing runs with varying cross-correlation reference targets (depending upon observing season). Besides, CFHT and KPNO data were single order spectra that, together with the large line broadening of $31 \mathrm{Com}$, resulted in uncertainties for individual measurements of as high as $4-7 \mathrm{~km} \mathrm{~s}^{-1}$. This is simply because radial velocity determinations with the cross-correlation technique from short chunks of the spectrum are intrinsically much less precise than from echelle spectra with a large wavelength coverage. Additionally, some of the cross-correlation profiles showed asymmetries that most likely resulted from a nonuniform distribution of star spots at certain epochs, e.g., in 1993 (Strassmeier et al. 1994).

Bounatiro (1993) listed a single radial-velocity measure of $-1.48 \mathrm{~km} \mathrm{~s}^{-1}$ in agreement with other mostly single measures, e.g., of $-1.25 \pm 4.11 \mathrm{~km} \mathrm{~s}^{-1}$ (deMedeiros \& Mayor 1999), $-0.7 \pm 2.8 \mathrm{~km} \mathrm{~s}^{-1}$ (Holmberg et al. 2007) or $-2.1 \pm 0.8$ (Gontcharov 2006). Odenkirchen et al. (1998) concluded on an average cluster velocity of $-0.1 \pm 0.2 \mathrm{~km} \mathrm{~s}^{-1}$ for Melotte 111 . Recently, Mermilliod et al. (2008) improved that value to $+0.01 \pm 0.08 \mathrm{~km} \mathrm{~s}^{-1}$ based on 28 members. Madsen et al. (2002) presented an astrometric radial velocity of $+2.1 \mathrm{~km} \mathrm{~s}^{-1}$ obtained from a kinematic solution of the Coma Berenices moving cluster using data from the Hipparcos main catalogue. The 40 members of the cluster in their study had a range of radial velocities between $+(1.2-2.8) \mathrm{km} \mathrm{s}^{-1}$. Our late 2007 and all 2008 STELLA spectra show a mean (barycentric) velocity of $+0.56 \pm$ $0.43(\mathrm{rms}) \mathrm{km} \mathrm{s}^{-1}$. Its rms is a combination of the rotational modulation and the uncertainty from the large rotational line broadening. The initial 2007 STELLA data had a zero-point offset with respect to the late 2007 and all 2008 data of $-0.35 \mathrm{~km} \mathrm{~s}^{-1}$ which was caused by a misalignment of the fiber exit with respect to the spectrograph collimator (see Weber et al. 2008) and was properly corrected. We have shifted the earlier 2007 data by $+0.35 \mathrm{~km} \mathrm{~s}^{-1}$. Note that the rms is also two times higher for the initial 2007 data than for the rest. A preliminary zero point from four K0III standard stars (Strassmeier et al. 2010) ties the STELLA velocities to the CORAVEL system when subtracting $460 \mathrm{~m} \mathrm{~s}^{-1}$ from the STELLA radial velocities. The mean radial velocity of 31 Com becomes then $+0.10 \pm 0.33 \mathrm{~km} \mathrm{~s}^{-1}$.

\subsubsection{Rotational modulation}

Starspots influence the symmetry of spectral line profiles and thereby influence also the radial velocity measurement. 
In eclipsing binaries such modulations are known as the Rossiter-McLaughlin effect when one component eclipses parts of one hemisphere of the other component which leads to an asymmetric line profile. This way stellar rotation was discovered in the early twenties of the previous century (Rossiter 1924; McLaughlin 1924). The effect is now being used to detect and characterize the transits of extrasolar planets (e.g. Dreizler et al. 2009). Radial velocity modulations due to the asymmetric line profiles of cool starspots can nowadays be detected, e.g., for the pre-main sequence star LkCa 19 (Huerta et al. 2008) or the very active main-sequence star V889 Her (Huber et al. 2009).

For a search of rotationally induced radial-velocity modulations in 31 Comae, we decided not to combine the various data sets, as with the APT photometry, and therefore refrained from an uncertain inter-annual, inter-instrumental zero-point correction. Instead, we focus on the best sampled, most homogeneous and most precise data set from the STELLA robotic telescope.

A phase-dispersion diagram of the 2008 data revealed a minimum at 6.80 days with a full amplitude of $\approx 250 \mathrm{~m} \mathrm{~s}^{-1}$. A CLEANed periodogram suggested a frequency peak corresponding to 6.73 days and comparable amplitude. None of the four algorithms applied (CLEAN, L-S, PDM, and DFT) showed a statistically truly significant peak though, partly due to the limited number of measurements available. Table 4 summarizes all results. DFT showed no clear single peak at all while PDM, CLEAN and L-S gave the highest peak around $f_{0} \approx 0.148 \mathrm{c} / \mathrm{d}$ (Fig. 5a). To assess the relevance of this frequency we applied a bootstrapping procedure to our data following the recipe of Press et al. (2002). One particular problem with this technique for period-search applications is the inherent change of the sampling. Therefore, only very robust results can be drawn from such an analysis which is, in a certain way, also its advantage. We generated one thousand test sets by replacing $36.8 \%(1 / e)$ of the original data with randomly chosen data points from the same data set. L-S and CLEAN where then applied to all thousand data sets and the five highest frequency peaks recorded for each. For both period analyses, L-S and CLEAN, the most frequently occurring peak was $0.148 \mathrm{c} / \mathrm{d}$ in $50 \%$ of the L-S cases and in $68.5 \%$ of the CLEAN cases. The second most dominant peak at $0.242 \mathrm{c} / \mathrm{d}$ was found in $46.7 \%$ of the L-S cases but only in $39.7 \%$ of the CLEAN cases. We consequentially used the average frequency as the starting value for a least-square minimization of a purely harmonic fit to our original velocities. It yielded a final peak of $0.14791 \mathrm{c} / \mathrm{d}$ that was only marginally different from the original frequency but with a full amplitude of $270 \pm 30 \mathrm{~m} \mathrm{~s}^{-1}$. The corresponding period of $6.761 \mathrm{~d}$ was used to finally phase the STELLA data as shown in Fig. 5b.

Saar \& Donahue (1997) and Hatzes (2002) gave a relationship of velocity amplitude $A$ in $\mathrm{m} \mathrm{s}^{-1}$ with spot coverage $F$ in per cent and line broadening $v \sin i$ in $\mathrm{km} \mathrm{s}^{-1}(A=(8.6 v \sin i-$ 1.6) $\left.F^{0.9}\right)$. This relationship would suggest $F \approx 40 \%$ for $31 \mathrm{Com}$. We conclude, firstly, that the star is single and that the 6.8-day period is indeed the true rotation period and, secondly, that the spot and/or surface-velocity distribution was significantly asymmetric with respect to the central meridian so that the diskintegrated radial velocities could be modulated.

\section{Global physical parameters of $\mathbf{3 1}$ Comae}

\subsection{Variability time scale}

Figure 4 shows phase plots of the MOST data as well as of our best-sampled APT T6 data. The STELLA radial velocities are shown in Fig. 5b. For all cases the peak-to-peak amplitudes
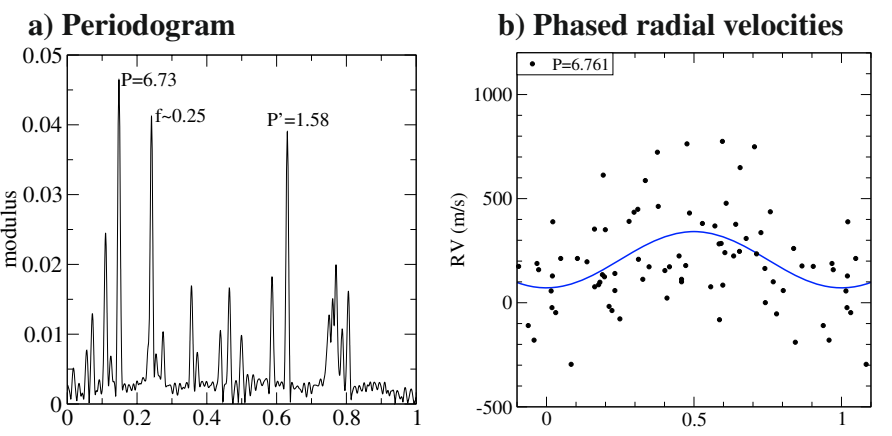

Fig. 5. STELLA radial velocities from the $2007 / 2008$ observing season. a) CLEAN periodogram; b) phased radial-velocity curve. None of the four periodogram algorithms, CLEAN, L-S, PDM, and DFT, showed a statistically truly significant peak. However, a bootstrapping procedure identifies the $f=0.148 \mathrm{c} / \mathrm{d}$ peak as the most solid frequency. This peak is then used as a starting value for a least-square minimization of a purely harmonic function and results in the best-fit period of $6.761 \mathrm{~d}$. The sinusoidal peak-to-peak amplitude is $270 \mathrm{~m} \mathrm{~s}^{-1}$.

are at the mmag or the $\mathrm{m} \mathrm{s}^{-1}$ level. The MOST data are plotted with both possible periods (3.38 and 6.80 days, respectively). The 3.38-day period gives a slightly larger amplitude but increases the overall scatter by approximately $20 \%$. The 6.8 -day period preserves the scatter as evident in the time plot in Fig. 1a but makes the phased light curve more complex. Nevertheless, the MOST light curve resembles that of the Sun during sunspot maximum (see, e.g., Fröhlich 2009), being likely dominated by bright faculae rather than dark spots. Changes of the light-curve shape from one rotation to the next suggests surface variability on time scales shorter than the rotational period. In white light, like for MOST, it stems from a mixture of spots and faculae and presumably is dominated by faculae that have their emission peak in the blue part of the spectrum. This is likely the reason why it is so difficult to pinpoint the rotation period of Hertzsprung-gap giants ${ }^{1}$. We may add though that we never saw a flare in our long-term $B V R I$ photometric monitoring.

\subsection{Distance, radius, rotational velocity, and inclination}

Bell \& Gustafsson (1989) predicted an angular diameter of 0.91 mas based on the apparent bolometric flux of $30.5 \times$ $10^{-15} \mathrm{~W} \mathrm{~cm}^{-2}$. It is also listed in the CHARM-2 catalog (Richichi et al. 2005). An independent estimate for future VLTI measurements by Wittkowski et al. (2002) arrived at the same diameter. Hipparcos originally measured a trigonometric parallax of $10.62 \pm 0.90$ mas (ESA 1997). The revised Hipparcos parallax of $11.22 \pm 0.23$ mas (van Leeuwen 2007) corresponds now to a distance of $89.1_{-1.7}^{+1.9} \mathrm{pc}$ instead of $94_{-7}^{+9} \mathrm{pc}$. Both parallaxes are in agreement with estimated parallaxes from a kinematic (open-cluster) solution by Madsen et al. (2002) though. The new parallax and the angular diameter convert to a stellar radius of $8.74 \pm 1.1 R_{\odot}$ for $31 \mathrm{Com}$, where the error includes an estimated $10 \%$ error for the angular diameter.

Earlier $v \sin i$ measurements based on photographic plates and partly on low spectral resolution and moderate $S / N$ ratio ranged from $90 \mathrm{~km} \mathrm{~s}^{-1}$ to $50 \mathrm{~km} \mathrm{~s}^{-1}$ (Bernacca 1973; see also Wallerstein et al. 1994). More modern measures gave generally smaller values, e.g., $57.0 \pm 1.5 \mathrm{~km} \mathrm{~s}^{-1}$ (Strassmeier et al. 1994), $66.7 \pm 6.6 \mathrm{~km} \mathrm{~s}^{-1}$ (deMedeiros \& Mayor 1999), and

\footnotetext{
1 The same is true for disk-integrated white-light data of the Sun, as demonstrated, e.g., from the ACRIM data by Strassmeier \& Oláh (2004).
} 
$63.0 \pm 3.2 \mathrm{~km} \mathrm{~s}^{-1}$ (Reiners 2006). With the higher resolution STELLA spectra and their much broader wavelength range, combined with the more direct synthetic spectrum fitting as described in Sect. 4.3, we revise our earlier $v \sin i$ measurement to $67 \pm 2 \mathrm{~km} \mathrm{~s}^{-1}$ (with a fixed microturbulence of $1.5 \mathrm{~km} \mathrm{~s}^{-1}$ and a macroturbulence of $5 \mathrm{~km} \mathrm{~s}^{-1}$ ). Note that Bernacca (1973) already listed a measurement of $67 \pm 3 \mathrm{~km} \mathrm{~s}^{-1}$.

With this $v \sin i$ and a period of $6.761 \mathrm{~d}$, the minimum radius, $R \sin i$, is $8.95 \pm 0.27 R_{\odot}$. Despite it is larger than the radius from the angular diameter, the two values are consistent within their errors. We conclude that the stellar inclination, $i$, is most likely close to $90^{\circ}$, i.e. equator-on. However, the uncertainties of the angular diameter based radius and the $v \sin i$ measure formally allow an inclination of as low as $\approx 60^{\circ}$.

\subsection{Effective temperature, gravity, and metallicity}

Gray et al. (2001a,b) had 31 Com in their sample of lateA to early-G stars. They fitted low-resolution spectra in the 380-460 nm range with synthetic spectra based on ATLAS-9 atmospheres simultaneous with fluxes from Strömgren photometry and found $T_{\text {eff }}=5810 \mathrm{~K}, \log g=3.42$, a "microturbulence" of $1.7 \mathrm{~km} \mathrm{~s}^{-1}$, and a metallicity of $-0.27 \mathrm{dex}$ with respect to the Sun $^{2}$. Given its uncertainties of $\pm 80 \mathrm{~K}, \pm 0.10 \mathrm{dex}$ in $\log g$, and \pm 0.10 in metallicity, the values are in agreement with the $u v b y$ based Geneva-Copenhagen (GC) survey result of $5875 \mathrm{~K}$ but in disagreement with its original $[\mathrm{Fe} / \mathrm{H}]=+0.14$ value $($ Holmberg et al. 2007). The revised GC catalog (Holmberg et al. 2009) lists $T_{\text {eff }}=5533 \mathrm{~K}$ and $[\mathrm{Fe} / \mathrm{H}]=-0.17$. The infrared flux method was used by Bell \& Gustafsson (1989) to obtain $5841 \mathrm{~K}$. Other $T_{\text {eff }}$ values in the literature are, e.g., $5623 \pm 150 \mathrm{~K}$ (Reiners 2006) based on $u v b y \beta$ photometry or $5572 \mathrm{~K}$ (Böhm-Vitense 2004) based on the Tycho/Hipparcos $B-V$. The sub-solar metallicity of -0.27 dex, i.e. the corrected Gray et al. (2001a) value, is somewhat in disagreement with $31 \mathrm{Com}$ being a member of Melotte 111. Odenkirchen et al. (1998) compiled a mean cluster metallicity of -0.062 with a dispersion of 0.023 . We notice that the two other rapidly rotating, early-G giants in Table 1 in Gray et al. (2001b), $\psi^{3}$ Psc and 35 Cnc, have been measured with practically the same metallicity as $31 \mathrm{Com}$, as well as similar $E(b-y)$. This is particularly interesting because all three stars are very active and rapidly rotating giants, though their Strömgren fluxes likely not a good choice for reddening determinations.

We used five out of the 81 echelle orders of our STELLA-SES spectra to determine the effective temperature, gravity and metallicity by fitting a synthetic spectrum. This fit is shown in Fig. 6. We employ the method and the numerical tools described in Allende-Prieto et al. (2004, 2006). Synthetic spectra are tabulated with metallicities between -1 dex and +0.5 dex in steps of 0.5 dex, logarithmic gravities between 0 and 5 in steps of 0.5 , and temperatures between $3500 \mathrm{~K}$ and $50000 \mathrm{~K}$ in steps of $250 \mathrm{~K}$ for a wavelength range of $380-920 \mathrm{~nm}$. This grid is then used to compare with the five selected echelle orders of each spectrum (all in the wavelength range between 549-623 nm). The final values per spectral order are chosen on the basis of a weighted least-squares minimization fit with predetermined and fixed $v \sin i$. From a total of 187 spectra, the mean and the standard deviations comprise then the best value and its internal precision. We found $T_{\text {eff }}=5660 \pm 42 \mathrm{~K}, \log g=3.51 \pm 0.09$,

\footnotetext{
${ }^{2}$ For stars later than F5, Gray et al. (2001a) suggested corrections to the values listed in their Table 1 for $\log g$ of +0.12 , for $[\mathrm{Fe} / \mathrm{H}]$ of $+0.06 \mathrm{dex}$, and for microturbulence of $-0.5 \mathrm{~km} \mathrm{~s}^{-1}$, which has been applied here.
}

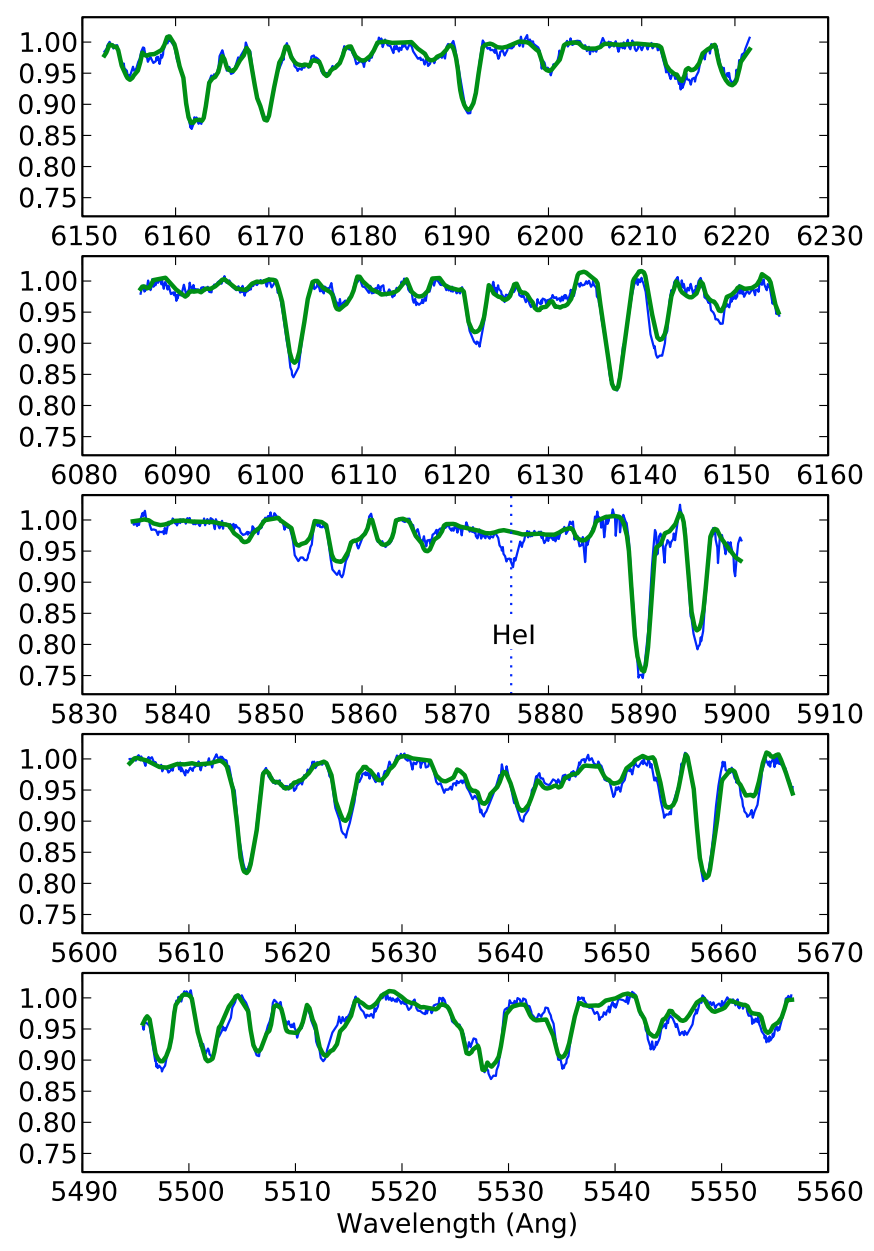

Fig. 6. Portions of five echelle orders from STELLA used to derive the photospheric parameters for $31 \mathrm{Com}$. The thin line is the data and the thick line is our synthetic-spectrum fit with PARSES. Note the "misfit" for the He I 587.56-nm line (total line equivalent width $W_{\lambda}=130 \mathrm{~m} \AA$; middle panel), indicating that the line is chromospheric in origin.

and $[\mathrm{Fe} / \mathrm{H}]=-0.15 \pm 0.03$. Our spectroscopic $T_{\text {eff }}$ value is significantly cooler than the Gray et al. (2001a,b) value but agrees with the Strömgren colors, while $\log g$ and the metallicity are better constrained and agree with the Gray et al. study within their respective error bars. Our metallicity tends to be more solar and now better agrees with the average Melotte-111 cluster metallicity.

Figure 6 also shows the detection of a He I D3-line at $587.56 \mathrm{~nm}$. The presence of this line indicates non-radiative heating processes in the atmosphere on 31 Comae since the line cannot be excited at photospheric temperatures. After subtraction of the photospheric fit (the thick line in Fig. 6) the blendcorrected equivalent width of the He I line is $95 \pm 5 \mathrm{~m} \AA$. This is exceptionally strong with respect to active $\mathrm{G}$ and $\mathrm{K}$ dwarfs (at least factor 2-3) in the sample of Saar et al. (1997) and again manifests the strong chromospheric magnetic activity of 31 Comae.

\subsection{Mass, luminosity, age, and angular momentum}

The distance of $89 \mathrm{pc}$ and the apparent $V$ brightness of 4.935 convert to an absolute magnitude of $+0 \mathrm{~m} .19 \pm 0.05$. The star is closer than $100 \mathrm{pc}$ and thus no interstellar absorption was taken into account despite the fact that Gray et al. (2001a) inferred $E(b-y)=0$ m 06 from Strömgren indices, but see above. With a 
K. G. Strassmeier et al.: Rotation and magnetic activity of the Hertzsprung-gap giant 31 Comae

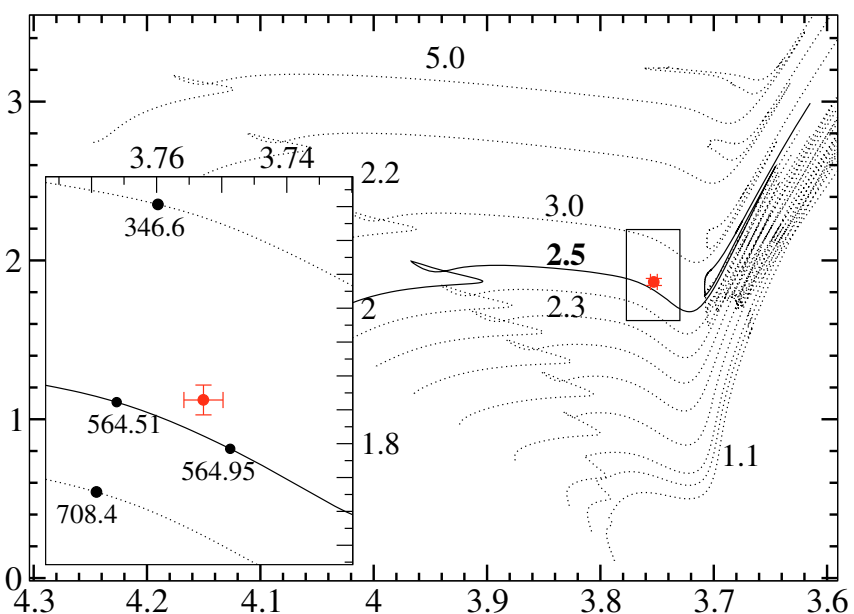

Fig. 7. 31 Comae in the H-R diagram $\log L / L_{\odot}\left(\log T_{\text {eff }}\right)$. Its position and observational uncertainties are indicated as a dot with bars. The tracks are from the grid of Pietrinferni et al. (2004) for a metallicity of $[\mathrm{Fe} / \mathrm{H}]=-0.15$ and were computed with overshooting. Tracks for various masses are indicated. The insert shows a zoom of the boxed region from which we directly infer a nominal mass of $2.6 \pm 0.1 M_{\odot}$ and an age of $\approx 540 \mathrm{Myr}$ for 31 Comae.

bolometric correction of -0.102 taken from Flower (1996) and based on $T_{\text {eff }}=5660 \mathrm{~K}$, or $B-V=0^{\mathrm{m}} 67$, a luminosity of $73.2 \pm 4 L_{\odot}$ follows (adopting $M_{\mathrm{bol}}=4.75$ for the Sun). If we deduce the radius from the predicted angular diameter, and use the same $T_{\text {eff }}$ as above, the luminosity is $77.7 \pm 13 L_{\odot}$, in fair agreement with the distance-based luminosity. The $42-\mathrm{K}$ error for $T_{\text {eff }}$ makes only little difference here. According to the evolutionary tracks from the BaSTI grid (Pietrinferni et al. 2004) with $[\mathrm{Fe} / \mathrm{H}]=-0.15$ and with overshooting the best match in the $\mathrm{H}-\mathrm{R}$ diagram is provided with a mass of $2.60 \pm 0.10 M_{\odot}$ and an isochrone with a nominal age of $540 \mathrm{Myr}$. Figure 7 shows the position of $31 \mathrm{Com}$ with respect to these evolutionary tracks. The insert is a zoom and indicates the aging sequence for a $2.5-M_{\odot}$ track. Note that the error bars that we give in Table 5 for the mass and the age are solely with respect to the observational uncertainties and do not take into account the uncertainties of the tracks themselves. Note also that a comparison with tracks without overshooting (and same metallicity as above) would increase the mass by $\approx 10 \%$ and decrease the age by $\approx 36 \%$.

The open-cluster age of Melotte 111 in the literature varies between $300 \mathrm{Myr}$ and $1 \mathrm{Gyr}$ but recent isochrone fittings constrain an age between 400-500 Myrs (Odenkirchen et al. 1998; see also the summary in Casewell et al. 2006). Our new value is more precise and likely also more accurate and agrees well with the age of $0.5 \pm 0.1 \mathrm{Gyr}$ listed in the revised GC catalog of Holmberg et al. (2009).

The absolute parameters in Table 5 constrain the logarithmic (pseudo) angular momentum, $m R v$, to $2.92 \pm 0.05$ with respect to today's Sun. This appears larger than expected from simple conservation arguments (Gray 1992). According to the Pietrinferni et al. (2004) models, 31 Com was a B9 star on the ZAMS with $T_{\text {eff }} \approx 11750 \mathrm{~K}$ and a radius of $\approx 2 R_{\odot}$. If we assume mass and angular-momentum conservation its equatorial rotational velocity was then around $176 \mathrm{~km} \mathrm{~s}^{-1}$, which is faster by $\approx 20 \%$ than an average B9-A0V star (Gray 1992).

With the current values for 31 Com and their errors given, can we validate or falsify the usual assumption of angularmomentum conservation from the ZAMS until today? Just recently, Kim \& Barnes (2008) computed evolutionary tracks and moment-of-inertia models for a $2.5 M_{\odot}$ star using the Yale
Table 5. Astrophysical properties of 31 Comae.

\begin{tabular}{lll}
\hline \hline Parameter & Value & Based on \\
\hline$V_{0}$, mag & 4.935 & APT $V$ photometry \\
Distance, pc & $89.1_{-1.7}^{+1.9}$ & revised Hipparcos \\
$M_{V}$ & $+0.19 \pm 0.05$ & from $V_{0}$ and distance \\
Classification, MK & G0III & Gray et al. (2001a) \\
Temperature, K & 5810 & Gray et al. $(2001 \mathrm{a})$ \\
& $5660 \pm 42$ & STELLA spectra \\
Radius, $R_{\odot}$ & $8.74 \pm 1.1$ & angular diameter \\
Minimum radius, $R_{\odot}$ & $8.95 \pm 0.27$ & from $P_{\text {rot }} \&$ sin $i$ \\
Luminosity, $L_{\odot}$ & $73.2 \pm 4$ & from $M_{V}$ and B.C. \\
& $77.7 \pm 13$ & from $R$ and $T_{\text {eff }}$ \\
Gravity, cgs & 3.42 & Gray et al. $(2001 \mathrm{a})$ \\
& $3.51 \pm 0.09$ & STELLA spectra \\
Mass, $M_{\odot}$ & $2.6 \pm 0.1$ & evolutionary tracks \\
Age, Myr & $540_{-21}^{+24}$ & isochrone fitting \\
Metallicity, $[\mathrm{M} / \mathrm{H}]$ & -0.21 & Gray et al. $(2001 \mathrm{a})$ \\
& -0.17 & GC, Strömgren phot. \\
& $-0.15 \pm 0.03$ & STELLA spectra \\
Li abundance, $H=12$ & $3.0 \pm 0.15$ & STELLA spectra \\
Average $\mathrm{RV}, \mathrm{km} \mathrm{s}{ }^{-1}$ & $+0.10 \pm 0.33$ & STELLA spectra \\
$v$ sin $i, \mathrm{~km} \mathrm{~s}^{-1}$ & $67 \pm 2$ & STELLA spectra \\
Rotation period, days & $6.80 \pm 0.06$ & MOST photometry \\
& $7.04 \pm 0.12$ & APT $y$ photometry \\
Inclination, deg & $6.76 \pm 0.02$ & STELLA radial velocity \\
\hline
\end{tabular}

Notes. Values not cited in the third column were obtained in this paper. $\mathrm{GC}=$ Geneva-Copenhagen survey.

Rotating Star Evolution Code (Demarque et al. 2008) with solar metallicity. In their models, a convective envelope first appears at $539 \mathrm{Myr}$, so no mass loss due to magnetized winds is expected before that. The moment of inertia of such a model at the ZAMS, i.e. 3.8 Myr post the birthline, is $4.9 \times 10^{54} \mathrm{~g} \mathrm{~cm}^{2}$. It decreases briefly at 5-10 Myr to a minimum of $3.7 \times 10^{54} \mathrm{~g} \mathrm{~cm}^{2}$, then increases steadily so that at $540 \mathrm{Myr}$ it is $5.8 \times 10^{55} \mathrm{~g} \mathrm{~cm}^{2}$. At this last stage, the model turns out a star that is at $5570 \mathrm{~K}$ and $35.6 L_{\odot}$. As this is discrepant to our measured values by about $90 \mathrm{~K}$ for the temperature and a factor of two for the luminosity, Kim \& Barnes (priv. commun.) recomputed the $2.5 M_{\odot}$ model including core overshoot of 0.2 and with sub-solar metallicity. The results show a luminosity of $80.5 L_{\odot}$ and $T_{\text {eff }}=5794 \mathrm{~K}$ at an age of $540.8 \mathrm{Myr}$, which now seems to agree with our observations. The moment of inertia is $5.3 \times 10^{55} \mathrm{~g} \mathrm{~cm}^{2}$ at this age, having increased from $3.3 \times 10^{54} \mathrm{~g} \mathrm{~cm}^{2}$ at the ZAMS by more than an order of magnitude. Consequently, our rotation period of $6.8 \mathrm{~d}$ at $540.8 \mathrm{Myr}$ works out to an expected period of $0.42 \mathrm{~d}$ on the ZAMS, corresponding essentially to maximal angular momentum of the model, and hence no loss by today's age of $31 \mathrm{Com}$. At the base of the giant branch at $541.7 \mathrm{Myr}$, and $5400 \mathrm{~K}$ and $56 L_{\odot}$, the moment of inertia will be $1.69 \times 10^{56} \mathrm{~g} \mathrm{~cm}^{2}$, so the expected rotation period in $\approx 1$ Myr would be $\approx 22 \mathrm{~d}$ if no further angular-momentum loss takes place. This translates to a (linear) period decrease $\mathrm{d} P / \mathrm{d} t=4 \times 10^{-7}$ which is, unfortunately, not detectable with the current precision.

The above moment-of-inertia model with core overshoot is the very first one computed, so it is currently only safe to say that angular momentum loss via winds has not taken place despite the star having crossed much of the Hertzprung gap. This is entirely consistent with stars starting with maximal or nearmaximal angular momentum on the ZAMS and conserving it up to and throughout most of the Hertzsprung gap. We also assumed rigid-body rotation throughout the age of $31 \mathrm{Com}$ and in particular also when on the ZAMS and shortly thereafter when 
the moment of inertia is at a minimum. It is remarkable that the predicted onset of envelope convection coincides so precisely with the current age of $31 \mathrm{Com}$. With the further increase of the depth of the convective envelope towards the RGB the star will likely maintain its magnetic activity until it arrives at the end of its first ascend of the RGB when significant mass loss sets in. However, with the increase of dynamo activity due to the increase of the convective envelope, $31 \mathrm{Com}$ must be also prone to magnetic braking, comparable to solar-mass main-sequence stars as we know it from the Skumanich law (Skumanich 1972; see also e.g. Barnes 2003). The predicted period decrease becomes then just a lower limit.

\subsection{Lithium abundance}

Our high-resolution spectra show a strong Li I resonance line at $670.78 \mathrm{~nm}$, as already found by Wallerstein et al. (1994), Böhm-Vitense (2004), and others. The only other lithium line in the optical spectrum, the subordinate line at $610.36 \mathrm{~nm}$ (actually a close triplet), appears fully blended with the nearby $\mathrm{Ca}$ I+Fe I blend at $610.27 \mathrm{~nm}$ and remains practically unmeasurable. The de-blended equivalent width of the $670.78 \mathrm{~nm}$ line is $171 \pm 20 \mathrm{~m} \AA$. It is blended in the blue wings with the unresolved Fe I+V I line pair at $670.5 \mathrm{~nm}$, itself having an equivalent width of $96 \mathrm{~m} \AA$ from a double Gaussian fit, and in the red wing by several unresolved $\mathrm{CN}$ and ${ }^{24} \mathrm{MgH}$ features. Note that the nearby $\mathrm{Ca}$ I line at $671.7 \mathrm{~nm}$ appears slightly stronger than the $\mathrm{Li}$ line with an equivalent width of $\approx 188 \mathrm{~m} \AA$. The $171-\mathrm{m} \AA$ equivalent width of $\mathrm{Li}$ is converted to an abundance of $\log n(\mathrm{Li}) \approx 3.0$ (with respect to $\log n(\mathrm{H})=12$ ) based on $T_{\text {eff }}=5660 \mathrm{~K}$ and $\log g=3.5$ in the NLTE tables in Pavlenko \& Magazzú (1996). Due to the large $v \sin i$ we cannot separate the two isotopes ${ }^{6} \mathrm{Li}$ and ${ }^{7} \mathrm{Li}$. The uncertainties of $\pm 42 \mathrm{~K}$ for $T_{\text {eff }}$ and $\pm 20 \mathrm{~m} \AA$ for the equivalent width convert into an uncertainty of the combined isotope abundance of \pm 0.15 dex. 31 Comae is therefore still a Li-rich giant close to the meteoritic abundance (see, e.g., Charbonnel \& Balachandran 2000), but the start of the Li dilution for a $2.6-M_{\odot}$ star is predicted to occur nearly exactly at its current age (Charbonnel \& Balachandran 2000). However, given the uncertainties of the tracks, the star's subsolar metallicity, and the observational uncertainties, we may just state that $31 \mathrm{Com}$ is near the onset of Li dilution.

\section{Doppler imaging}

\subsection{Method and assumptions}

Doppler imaging is an inverse problem that amounts to solving the integral equation relating the surface temperature distribution to the observed line profiles while controlling the effects of noise in the data through a regularizing functional (e.g. Piskunov \& Rice 1993). For 31 Com, we employ our new Doppler-imaging code iMAP (Carroll et al. 2007) coupled with a data de-noising method based on a principal component analysis (PCA) as implemented by Carroll et al. (2009) and Kopf et al. (2009). In this approach a set of local line profiles is decomposed into their respective eigenspectra and an artificial neural network is trained to approximate the non-linear mapping between atmospheric parameters and principal components. A total of 1487 individual spectral lines in the wavelength range 480-860 nm were used for the de-noising of the 31 Comae time series of three selected spectral lines; Fe I 549.7516 nm (excitation energy of $1.011 \mathrm{eV}$, transition probability $\log g f=-2.849)$, Fe I $641.1659 \mathrm{~m}(3.654 \mathrm{eV}, \log g f=-0.595)$, and Ca I $643.9075 \mathrm{~nm}$
(2.526 eV, $\log g f=+0.47)$. Only lines with a residual depth of greater than 0.2 with respect to the continuum are selected.

The basic free parameter of our PCA de-noising method is the number of principal components. No strict rule can be given for the choice of this value but we followed the strategy laid out in Martínez González et al. (2008). The procedure suggests 9 components for the Fe I 549.7-nm and Ca I 643.9-nm profiles and 15 components for Fe I $641.1 \mathrm{~nm}$. Blending line lists for the three main mapping lines were extracted from VALD (see Heiter et al. 2007). The full spectrum was then synthesized in the range from -6 to $+6 \AA$ around the line center of the three main mapping lines using the program SPECTRUM (Gray 2000). In the case of Fe I 5497 there is a total of 46 blends including some weak molecular lines, mostly from $\mathrm{TiO}$ and $\mathrm{VO}$. The inversion is performed from the central $3 \AA$ on the basis of a $6^{\circ} \times 6^{\circ}$ surface grid, while the radiative transfer calculations are done with a $2^{\circ} \times 2^{\circ}$ grid.

A grid of model atmospheres with temperatures between $T_{\text {eff }}=3750 \mathrm{~K}$ and $6750 \mathrm{~K}$ in steps of $250 \mathrm{~K}$ and fixed $\log g=3.5$ were taken from the ATLAS9 CD (Kurucz 1993). For each model atmosphere local line profiles were computed under the assumption of solar abundances and a microturbulence of $1.7 \mathrm{~km} \mathrm{~s}^{-1}$ according to the analysis of Gray et al. (2001a). Note that the model atmospheres themselves are based on a microturbulence of $2 \mathrm{~km} \mathrm{~s}^{-1}$. Gray (1989) gives a typical radialtangential macroturbulence velocity of $5 \mathrm{~km} \mathrm{~s}^{-1}$ for a $\mathrm{G} 0$ giant which we also adopted here.

\subsection{Data}

STELLA observed 31 Com 37 times on 11 consecutive nights in March 10-22, 2008. Two nights were lost due to bad weather. An integration time of 800 s resulted in $S / N$ ratios between 180-300:1 depending on atmospheric conditions and target hour angle. The PCA line profiles reach $S / N$ ratios of 1100:1 (gain factor of 7.5). These spectra are phase folded with the ephemeris

$H J D=2450000+6.761 \times E$,

where the zero epoch is arbitrary and the period is our period from the radial-velocity variations (Sect. 3.4.2) because it seems better established than the photometric periods. As for the MOST data we combined two consecutive stellar rotations under the assumption that the intrinsic stellar surface structure did not change. Although a risky assumption it is the only way to gain full phase coverage without significant phase gaps. Simulations had shown that the ambiguities in the recovery induced by phase gaps can be of much larger scale than small intrinsic changes (Rice \& Strassmeier 2000). Their smearing within the line profiles due to the finite integration time is negligible for $31 \mathrm{Com}$ though.

Bumps in the absorption line profiles of 31 Comae were already noticed in our few CFHT spectra in 1993 (Strassmeier et al. (1994). In 31 Com, these bumps appear two to three times weaker when compared to line profiles of spotted RS CVn stars and barely reach $1 \%$ of the continuum. This is due to the combination of the high photospheric temperature that gives less contrast to cool spots and the large line broadening that makes the profile prone to blending. Doppler imaging of $31 \mathrm{Com}$ is therefore challenging. 
a) $\mathrm{Fe}$ I $549.7 \mathrm{~nm}$
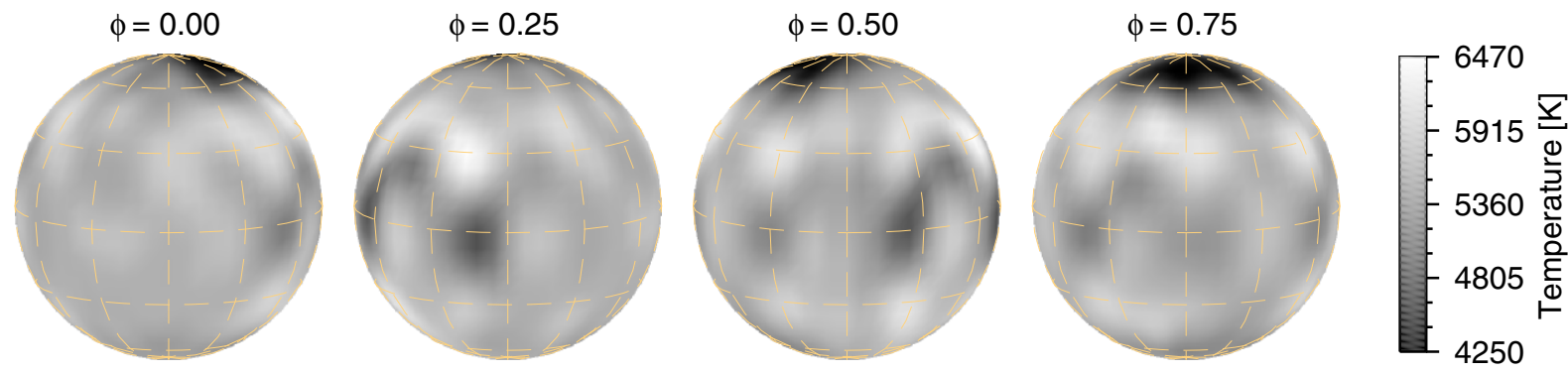

b) Fe I $641.1 \mathrm{~nm}$
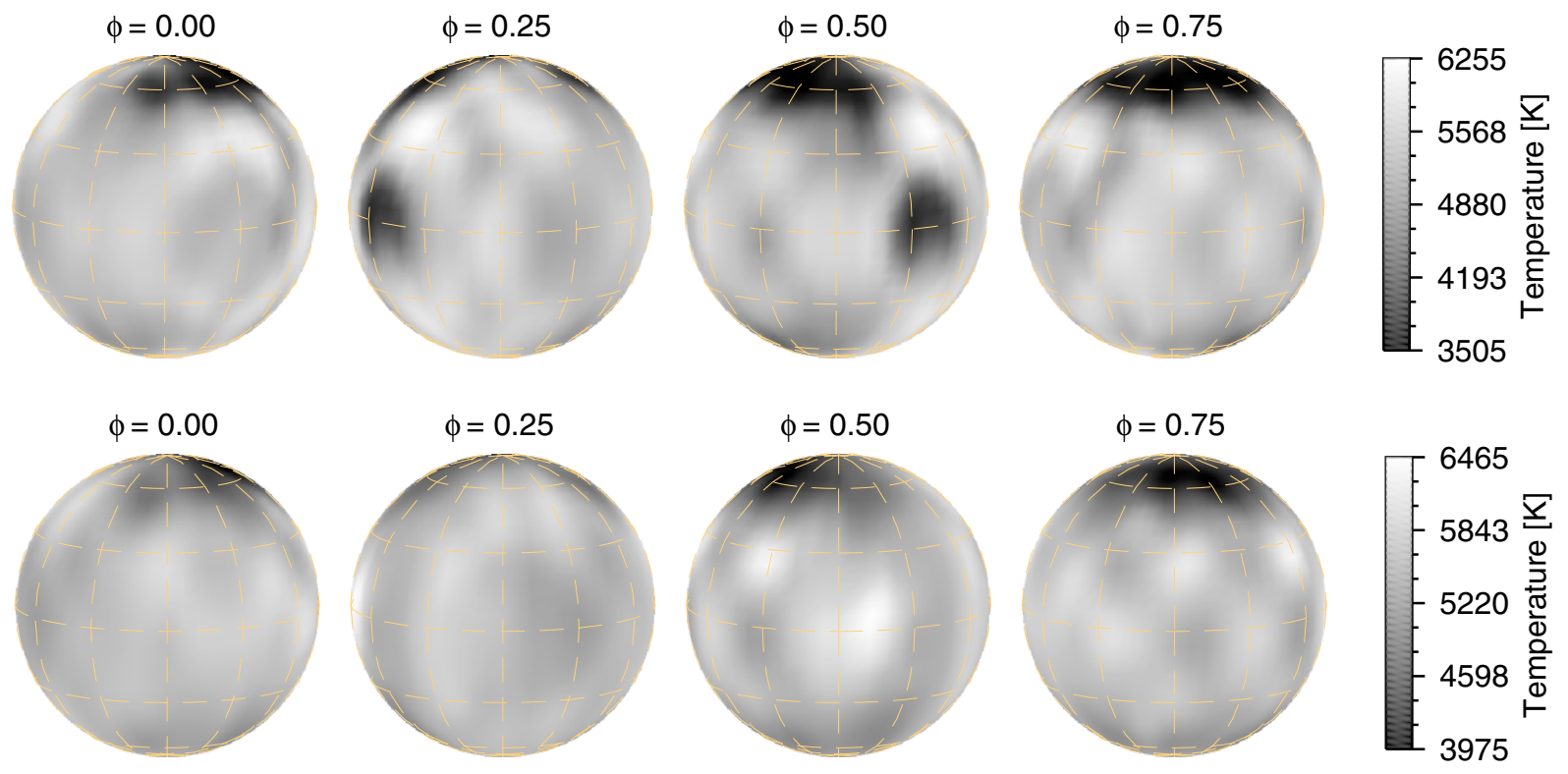

c) $\mathrm{Ca}$ I $643.9 \mathrm{~nm}$

Fig. 8. Orthographic temperature Doppler images of 31 Com inverted, from top to bottom, from a) the Fe I 549.7-nm line, b) Fe I $641.1 \mathrm{~nm}$, c) Ca I $643.9 \mathrm{~nm}$. A total of 1487 spectral lines were used to denoise the individual spectral lines. The color code represents temperature as indicated. A cool polar cap is reconstructed from all lines with an average temperature difference with respect to the effective temperature of $1600 \mathrm{~K}$. Several small warm features, also with respect to the effective temperature, were reconstructed at low to mid-equatorial latitudes. Due to the large inclination of the rotational axis of $\approx 80^{\circ}$, the reconstructed polar spot must not be exclusively at the one visible rotational pole but might be more symmetric and could also cover the other (mostly) hidden pole.

\subsection{Results}

The error function evaluated during the inversion is defined as

$E=\frac{1}{(n-1)} \sum_{\phi} \sum_{\lambda}(O-C)^{2}$,

with $\phi=35$ rotation phases and $\lambda$ wavelength points per line profile ( $n=241$ for the Fe I 549.7-nm line and $n=281$ for the CaI 643.9 and Fe I 641.1 lines, respectively). This number of data points is such that the problem is essentially overdetermined and the least-squares fit predominates. We have 3.7 times as many profile points in total as we have pixels (2592) visible on the surface. Note that the pixel size is $6^{\circ} \times 6^{\circ}$. The fitting errors after 29 iterations on average were $\sim 1 \times 10^{-5}$ for Fe I $549.7 \mathrm{~nm}, \sim 8 \times 10^{-5}$ for Ca I $643.9 \mathrm{~nm}$, and $\sim 8 \times 10^{-6}$ for Fe I $641.1 \mathrm{~nm}$, corresponding to $S / N$ ratios between $316-354$. The ideal fit would be achieved with a $S / N$ ratio that matches the $S / N$ ratio of the de-noised time series, i.e. on average 1100:1. As this is by far not achievable indicates that most of the remaining noise is not photon noise but of external origin, as discussed in Rice \& Strassmeier (2000).

Figure 8 shows the PCA reconstructed surface images from Fe I $549.7 \mathrm{~nm}, \mathrm{Fe}$ I $641.1 \mathrm{~nm}$ and Ca I $643.9 \mathrm{~nm}$. The line-profile fits are shown in Fig. 9. The final images were reconstructed with a fixed inclination of the stellar rotational axis of $80^{\circ}$, i.e. close to equator-on. Such an inclination causes intrinsic problems for Doppler imaging because the algorithm cannot uniquely decide to which hemisphere it must assign the spots. This "mirroring effect" is well known in Doppler imaging and had been documented and simulated in the past (e.g. Vogt et al. 1987; Piskunov \& Wehlau 1994; Rice \& Strassmeier 2000). We cannot bypass it and must keep it in mind when interpreting the images. For example, the mirroring effect would contribute to a second polar spot and therefore allows no conclusion on whether there is indeed a second polar spot or not. Fortunately, Doppler images become rather insensitive to the actual choice of the inclination once $i \geq 70^{\circ}$.

Surprisingly, all spectral lines are reconstructed with a cool polar cap-like spot reaching down to mid latitudes of $\approx 60^{\circ}$. Additionally, warmer features appear grouped along an equatorial band of $\pm 30^{\circ}$ from the equator. The polar feature appears also structured in temperature and quite asymmetric. The features themselves are a solid reconstruction from the data but we could not say whether only one hemisphere has a polar spot or indeed both as indicated. The average spot temperature is reconstructed consistently from all spectral lines to be near $4050 \mathrm{~K}$, i.e. $\approx 1600 \mathrm{~K}$ cooler than the stellar effective temperature. The bright equatorial features are near $6000 \mathrm{~K}$, and thus $\approx 300-400 \mathrm{~K}$ 
a)

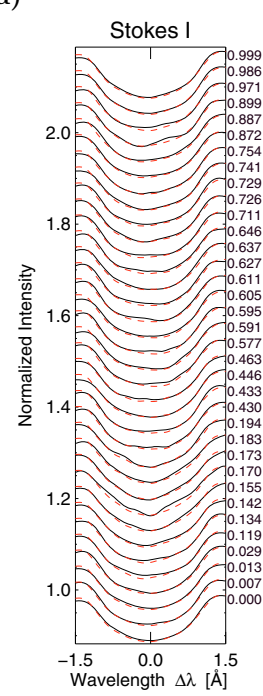

b)

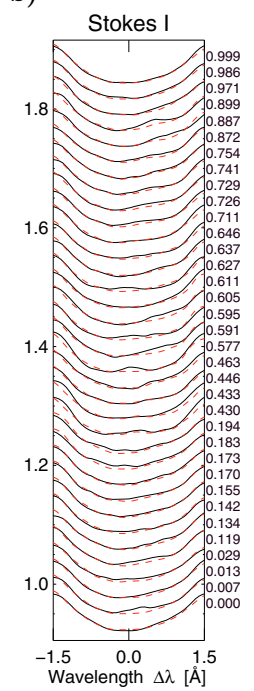

c)

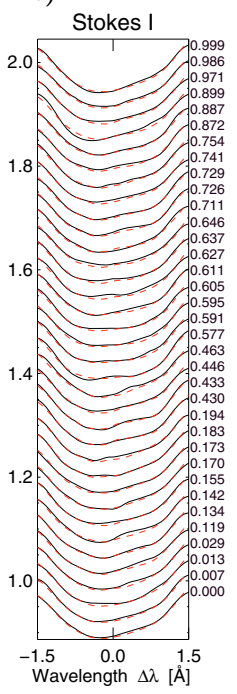

Fig. 9. Line profile fits. a) for the Fe I 549.7-nm line, b) the Fe I $641.1 \mathrm{~nm}$, and c) for the Ca I $643.9 \mathrm{~nm}$ line. The full lines are the fits and the dashed lines are the PCA-denoised observations. The numbers on the right side indicate rotational phase according to the ephemeris in Eq. (2).

warmer than the effective temperature. Such a temperature difference is significant for our mapping technique and the given quality of the PCA spectra (Kopf et al. 2009) but the location of the strongest of these features is located along an isoradial line with the equatorial cool spot at phase $\approx 0$ p. 4 and therefore bears the chance to be an artifact. We note though that the reconstruction from the strong $\mathrm{Ca}$ I line required a warm spot on the same surface location where the reconstruction from the other two lines recovered a cool spot and thus indicates that we may have resolved a chromospheric component in $\mathrm{Ca}$ I.

\section{Discussion}

Our Doppler images recovered a large cool polar spot and several small warm spots on the equator at the same time. The pure existence of a large polar spot is indeed puzzling for a star of that mass and size of the convective envelope. Because if flux tubes were located in a sub-adiabatic layer they would surface along a Coriolis-force dominated locus. A predicted thickness of the convective envelope of just 20\% (MESA code ${ }^{3}$; Paxton et al. 2010) is simply not sufficient for a significant deflection from radial direction. Therefore, flux tubes, if present at all, would surface at almost exactly the same latitude where they are being brought out of equilibrium and become buoyant (e.g. Schüssler \& Solanki 1992; Granzer et al. 2000). How they could even appear at the rotational pole remains to be explained. This leaves us with at least two very different interpretations. Firstly, either the flux tubes are generated already at the poles or were swapped up to the poles by a hypothetic, superstrong pole-ward meridional circulation (Holzwarth et al. 2007) or, secondly, the magnetic field that makes up the large polar cap is not of dynamo origin but of fossil origin like in Ap stars (e.g. Mathys 2007). The mass of $2.6 M_{\odot}$ and the solid argument that 31 Com was a very rapidly rotating $\mathrm{B} 9-\mathrm{A} 0$ star on the main sequence favors the latter interpretation.

Magnetic field measurements of evolved, early $\mathrm{G}$ giants are not only rare but also yielded comparably low field strengths

3 http://mesa. sourceforge.ne

(compared to late G giants). Hubrig et al. (1994) obtained a mean longitudinal component of the magnetic field of $49 \pm 6 \mathrm{G}$ on one night for the G1II bright giant $\epsilon$ Leo, while Plachinda (2005) obtained no statistically significant field strengths at all for $\alpha$ Aqr (G2Ib) and $\beta$ Aqr (G0Ib). Just recently, Auriére et al. (2008) measured a mean longitudinal field strength of between 20-100 G on the single G8 subgiant HR $1362=$ EK Eri. They infer a mean surface magnetic field of $270 \mathrm{G}$ from this. Although also a (late) G giant, EK Eri is different from $31 \mathrm{Com}$ because it is significantly less massive and it has already arrived at the end of the Hertzsprung gap where it became a very slow rotator now with a period of possibly 618 days (Dall et al. 2010). Nevertheless, the large-scale magnetic field was found to be dominated by a poloidal component, which is at least suggestive that this also may be the case for $31 \mathrm{Com}$.

A fossil magnetic field in the pre-main sequence stage has likely a decisive impact on the overall angular momentum with which the star appears on the ZAMS. Numerical simulations of pre main-sequence A stars show that the shrinking convective envelope pushes its magnetic field into the radiative core by turbulent pumping (e.g. Arlt 2009). At that time the magnetic quadrupole and non-axisymmetric modes already dominate the total magnetic energy. The field is then expected to enforce internal rigid rotation and the accompanying angular momentum rearrangement eventually leads to a rapidly rotating surface and a dredge up of, e.g., fresh lithium. This scenario is backed up by the observation that Li-rich early $\mathrm{F}$ giants are usually also rapidly rotating (Jasniewicz et al. 2006). When the star evolves across the Hertzsprung gap it will again develop a convective envelope and then start to dilute its surface lithium, as is also backed up by observations (de Laverny et al. 2003). To explain the Li dip, e.g., in the Hyades F dwarfs, Böhm-Vitense (2004) invokes a deep mixing process while the increase of the Li dip with age is suggested to be due to the decay of the fossil field. Recent measurements of the magnetic field of Ap stars in open clusters showed rms fields larger than about $1 \mathrm{kG}$ only when they are near the ZAMS (Landstreet et al. 2008). The time scale on which these large fields disappear varies strongly with mass, but was found to be about 250 Myr for 2-3 $M_{\odot}$ stars. It supports the picture that $31 \mathrm{Com}$ was already a magnetic star when on the main sequence.

On the contrary, for the Sun we have abundant evidence that the surface magnetic field and Ca II, UV and X-ray emission are all well correlated (see Schrijver \& Zwaan 2000, and references therein). The observation of X-ray emission and UV emission lines usually indicates a magnetically active star powered by a classical $\alpha \Omega$ interface dynamo. As 31 Com is a strong X-ray emitter with coronal loops peaking at a temperature near $10^{7} \mathrm{~K}$ (Scelsi et al. 2004) and with super rotationally broadened UV lines suggesting transient explosive activity in the chromosphere and transition region (Ayres et al. 2007), the question appears how could these be provided by a fossil predominantly poloidal magnetic field?

The Rossby number, $P_{\text {rot }} / \tau_{\mathrm{c}}$, has been accepted as indicating the efficiency of a classical dynamo, its convective turn-over time, $\tau_{\mathrm{c}}$, remains an elusive parameter though. It describes the ratio of a characteristic length of convection to a characteristic velocity, $\ell / u$, and is either determined theoretically (e.g. Kim \& Demarque 1996) or semi-empirically (e.g. Pizzolato et al. 2003). For 31 Com it was given by Gondoin (2005) as 50 days based on evolutionary calculations for a $2.2-M_{\odot}$ star with an effective temperature of $5320 \mathrm{~K}$. Thus, much longer than the rotational period measured. It would give a Rossby number of 0.13 and, according to the criterium of Durney \& Latour (1978), be by far sufficient 


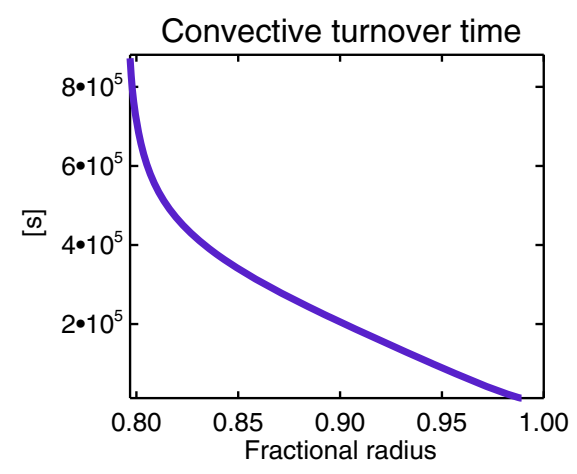

Fig. 10. The convective turn-over time, $\tau_{\mathrm{c}}$, in seconds as a function of depth in the convection zone of $31 \mathrm{Com}$. The adopted stellar model and its rotation period match our values in Table 5. Note that the depth of the convective envelope is $20 \%$ and the average turn-over time $\approx 5$ days.

to run an effective dynamo. We use the differential-rotation code of Küker \& Stix (2001) to recompute $\tau_{\mathrm{c}}$ as a function of depth based on its description through the pressure scale height, $H_{\mathrm{p}}$, and the classical mixing length, $\alpha$. The characteristic convective length is defined as $\ell=\alpha H_{\mathrm{p}}$, with $\alpha=2$. In the case of a fast rotator, the convective heat flux is no longer aligned with the temperature gradient and tilts towards the rotational axis. It then creates a horizontal heat flux from the equator to the poles (Küker \& Rüdiger 2008). Figure 10 shows the turn-over time for $31 \mathrm{Com}$ plotted versus the fractional stellar radius. The depth of the convection zone for a rotating $5660-\mathrm{K}$ giant is predicted to be $20 \%$ of the stellar radius and the average turn-over time approximately five days. The Rossby number is then above or near unity and an efficient dynamo would not be expected. We note that if 31 Com were a much slower rotator, say with a period of 700 days, the average turn-over time would be longer by approximately a factor two.

The puzzle can be resolved naturally when we assume that both types of magnetic field are present at the same time, i.e. a fossil, predominantly axisymmetric poloidal, field and a dynamo-generated, presumably non-axisymmetric equatorial, field. Then, the fossil field could be linked to the cool polar spot while the dynamo-generated field, based on a classical advection-driven dynamo in the convective envelope, would cause the equatorial warm spots and also the loops hosting the bulk of UV and X-ray emission. One could further speculate that the convective core of $31 \mathrm{Com}$ also runs a dynamo and that it interacts with the fossil field that resides in the radiative zone between the (convective) core and the shallow surface convection layer. Just recently, Featherstone et al. (2009) has demonstrated that the existence of a fossil field may induce a core dynamo to transit to such a strong-field dynamo at the edge of the convection zone. The induced field strengths may even be strong enough to cause flux emergence to the surface and then possibly may explain the observed polar spot. Such a spot could even manifest a new species in the zoo of starspots, not directly related to sunspots anymore (for a comparison see, e.g., Strassmeier 2009). The role of the magneto rotational instability and its relation to meridional circulation and differential rotation is yet to be considered and understood though and any conclusions just preliminary (see, e.g., Küker 2009).

\section{Summary and conclusions}

We have obtained the rotation period and other astrophysical parameters of the active G0 Hertzsprung-gap giant 31 Comae. The star appears to have just started its rotational braking phase towards the RGB. The rotation period of a Hertzsprung-gap giant constitutes an important constraint for models of the evolution of the angular momentum, the internal differential rotation and in particular for the mixing processes of light elements below the photosphere. It is also a crucial ingredient enabling Doppler imaging of stellar surface structure from spectral line profiles.

We conducted and analyzed photometric measurements from an 11-day campaign with the MOST satellite and found, for the first time, clear photometric variations with a likely period of either 3.38 days or $6.80 \pm 0.06$ days. This period is confirmed by our ground-based APT Strömgren data despite its significance alone being questionable due to the very small amplitudes at the mmag level. Precise radial velocities from highresolution echelle spectra were acquired over the course of two years with the STELLA robotic telescope as an independent data source. This revealed radial velocity variations with a period of $6.76 \pm 0.02$ days and a full amplitude of $270 \mathrm{~m} \mathrm{~s}^{-1}$ that we interpret to be the rotation period of the star. Together with the 6.80 -day MOST white-light period and the $\approx 7.0$-day APT photometric period, we are confident that we have found the true stellar rotation period. No other long-term velocity variations above the external precision of our spectra of $\approx 100 \mathrm{~m} \mathrm{~s}^{-1}$ (rms) were detected and we conclude that the star is indeed single.

Our Doppler images recovered a large cool polar spot. This is puzzling because, at the given location of $31 \mathrm{Com}$ in the $\mathrm{H}-$ $\mathrm{R}$ diagram, the predicted depth of the convective envelope is less than $20 \%$ of the stellar radius. Such a thin layer does not provide the volume to deflect flux tubes from an equatorial or mid-latitude sub-adiabatic layer beneath the convective envelope to the polar surface, according to models by, e.g., Schüssler \& Solanki (1992). We suggest that the underlying magnetic field near the rotational poles is not of a classical, envelope-dynamo origin but is of fossil origin and thus a remnant of a strong magnetic field from the star's main sequence years. It implies that we have mapped the atmospheric interaction with an axisymmetric poloidal component of this fossil magnetic field, resembling a dipole aligned with the rotational axis. Alternatively, the surface field at the poles could be made up of flux tubes that stem from a core dynamo that interacted with the fossil field and then got strong enough to become buoyant and traversed a much larger region of the star. On the contrary, the small and warmer features observed at equatorial latitudes are likely faculae as we know them from the solar analogy, suggesting a classical envelope-dynamo origin. It must be these faculae that also dominated the white light curve of our MOST photometry. We suggest that these faculae, together with the UV and X-ray emission, are caused by a classical envelope-interface dynamo that is predominantly bound to low-latitude regions.

Acknowledgements. K.G.S. and the AIP coauthors are grateful to the State of Brandenburg and the German federal ministry for education and research (BMBF) for their continuous support of the STELLA and APT activities. It is our pleasure to thank Sydney Barnes and Yong-Cheol Kim for computing the moment-of-inertia models for us. We particularly thank our anonymous referee whose comments resulted in a much improved paper. J.B.R., J.M.M., D.B.G., A.F.J.M., and S.M.R. are supported by funding from the Natural Sciences and Engineering Research Council (NSERC) Canada. R.K. is funded by the Canadian Space Agency. W.W.W. is supported by the Austrian Science Promotion Agency (FFG-MOST) and the Austrian Science Funds (FWF-P17580). The APT operation has been supported by grants from the FWF to M. Breger and KGS. This work has made use of BaSTI web tools, the MESA code and of the many CDS-Strasbourg services.

\section{References}

Allende-Prieto, C. 2004, AN, 325, 604

Allende-Prieto, C., Beers, T. C., Wilhelm, R., et al. 2006, ApJ, 636, 804 
Arlt, R. 2009, in Cosmic Magnetic Fields: From Planets, to Stars and Galaxies (Cambridge University Press), IAU Symp., 259, 443

Auriére, M., Konstantinova-Antova, R., Petit, P., et al. 2008, A\&A, 491, 499

Ayres, T. R., \& Linsky, J. E. 1980, ApJ, 241, 279

Ayres, T. R., Simon, T., Stern, R. A., et al. 1998, ApJ, 496, 428

Ayres, T. R., Hodges-Kluck, E., \& Brown, A. 2007, ApJS, 171, 304

Barnes, S. A. 2003, ApJ, 586, 464

Bell, R. A., \& Gustafsson, B. 1989, MNRAS, 236, 653

Bernacca, P. L. 1973, Contr. Oss. Astr. Univ. Padova Asiago, No. 294

Böhm-Vitense, E. 2004, AJ, 128, 2435

Bopp, B. W., \& Stencel, R. E. 1981, ApJ, 247, 131

Bopp, B. W., Dempsey, R. C., \& Maniak, S. 1988, ApJS, 68, 803

Bounatiro, L. 1993, A\&AS, 100, 531

Breger, M., Stich, J., Garrido, R., et al. 1993, A\&A, 271, 482

Carroll, T. A., Kopf, M., Ilyin, I., \& Strassmeier, K. G. 2007, AN, 328, 1043

Carroll, T. A., Kopf, M., Strassmeier, K. G., \& Ilyin, I. 2009, in Cosmic Magnetic

Fields: From Planets, to Stars and Galaxies (Cambridge University Press), IAU Symp., 259, 633

Casewell, S. L., Jameson, R. F., \& Dobbie, P. D. 2006, MNRAS, 365, 447

Charbonnel, C., \& Balachandran, S. C. 2000, A\&A, 359, 563

Dall, T. H., Bruntt, H., Stello, D., \& Strassmeier, K. G. 2010, A\&A, 514, A25

Demarque, P., Guenther, D. B., Li, L. H., Mazumdar, A., \& Straka, C. W. 2008, Ap\&SS, 316, 31

de Laverny, P., do Nascimento, J. D., Jr., Lébre, A., \& De Medeiros, J. R. 2003, A\&A, 410, 937

De Medeiros, J. R., \& Mayor, M. 1999, A\&AS, 139, 433

De Medeiros, J. R., do Nascimento, J. D., Sankarankutty, S., Costa, J. M., \& Maia, M. R. G. 2000, A\&A, 363, 239

Dreizler, S., Reiners, A., Homeier, D., \& Noll, M. 2009, A\&A, 499, 615

Durney, B. R., \& Latour, J. 1978, Geophys. Ap. Fluid Dynamics, 9, 241

ESA 1997, The Hipparcos and Tycho Catalogues, ESA SP, 1200

Featherstone, N. A., Browning, M. K., Brun, A. S., \& Toomre, J. 2009, ApJ, 705, 1000

Flower, P. J. 1996, ApJ, 469, 355

Fröhlich, C. 2009, A\&A, 501, L27

Gondoin, P. 1999, A\&A, 352, 217

Gondoin, P. 2005, A\&A, 444, 531

Gontcharov, G. A. 2006, Astron. Lett., 32, 759

Granzer, T., Schüssler, M., Caligari, P., \& Strassmeier, K. G. 2000, A\&A, 355, 1087

Granzer, T., Reegen, P., \& Strassmeier, K. G. 2001, AN, 322, 325

Gray, D. F. 1989, ApJ, 347, 1021

Gray, D. F. 1992, The observation and analysis of stellar photospheres, 2nd edn (Cambridge University Press)

Gray, R. O. 2000 http://www. phys. appstate. edu/spectrum

Gray, R. O., Napier, M. G., \& Winkler, L. I. 2001a, AJ, 121, 2148

Gray, R. O., Graham, P. W., \& Hoyt, S. R. 2001b, AJ, 121, 2159

Hagen, W., \& Stencel, R. E. 1985, AJ, 90, 120

Harris, D. L., \& Upgren, A. R. 1964, ApJ, 140, 151

Hatzes, A. P. 2002, AN, 323, 392

Heiter, U., Barklem, P., Fossati, L., et al. 2008, J. Phys., Conf. Ser., 130, 1, 012011

Holmberg, J., Nordstroem, B., \& Andersen, J. 2007, A\&A, 475, 519

Holmberg, J., Nordstroem, B., \& Andersen, J. 2009, A\&A, 501, 941

Holzwarth, V., Mackay, D. H., \& Jardine, M. 2007, AN, 328, 1108

Huerta, M., Johns-Krull, C. M., Prato, L., Hartigan, P., \& Jaffe, D. T. 2008, ApJ, 678,472

Huber, K. F., Wolter, U., Czesla, S., et al. 2009, A\&A, 501, 715

Hubrig, S., Plachinda, S. I., Hünsch, M., \& Schröder, K.-P. 1994, A\&A, 291, 890

Hünsch, M., Schmitt, J. H. M. M., \& Voges, W. 1998, A\&AS, 127, 251

Jasniewicz, G., Recio-Blanco, A., de Laverny, P., Parthasarathy, M., \& de Medeiros, J. R. 2006, A\&A, 453, 717

Kazarovets, E. V., Kireeva, N. N., Samus, N. N., \& Durlevich, O. V. 2003, IBVS 5422

Kim, Y.-C., \& Demarque, P. 1996, ApJ, 457, 340

Kim, Y.-C., \& Barnes, S. A. 2008, in The Art of Modeling Stars in the 21st Century (Cambridge Univ. Press), IAU Symp., 252, 117

Kopf, M., Carroll, T. A., \& Strassmeier, K. G. 2009, in Solar Polarization 5: In Honor of Jan Stenflo, ed. Berdyugina, S. V., Nagendra, K. N., \& Ramelli, R., ASP Conf. Ser., 405, 535

Korhonen, H., Berdyugina, S. V., Hackman, T., et al. 1999, A\&A, 346, 101

Korhonen, H., Berdyugina, S. V., Hackman, T., Strassmeier, K. G., \& Tuominen, I. 2000, A\&A, 360, 1067

Korhonen, H., Berdyugina, S. V., Tuominen, I., et al. 2001, A\&A, 374, 1049

Korhonen, H., Hubrig, S., Berdyugina, S. V., et al. 2009, MNRAS, 395, 282

Küker, M. 2009, in Proc. of the 15th Cambridge Workshop on Cool Stars, Stellar Systems and the Sun, AIP Conf. Proc., 1094, 77

Küker, M., \& Stix, M. 2001, A\&A, 366, 668
Küker, M., \& Rüdiger, G. 2008, in J. Phys., Conf. Ser., 118, 1, 012029

Kurucz, R. L. 1993, ATLAS-9, CD-ROM \#13

Lafler, J., \& Kinman, T. D. 1965, ApJS, 11, 216

Landstreet, J. D., Silaj, J., Andretta, V., et al. 2008, A\&A, 481, 465

Lockwood, G. W., Skiff, B. A., \& Thompson, D. T. 1993, in Stellar Photometry - Current Techniques and Future Developments, ed. C. J. Butler, \& J. Elliott, IAU Coll. 136 (Cambridge Univ. Press), 99

Lomb, N. R. 1976, Ap. Space Sci., 39, 447

Madsen, S., Dravins, D., \& Lindegren, L. 2002, A\&A, 381, 446

Martínez González, M. J., Asensio Ramos, A., Carroll, T. A., et al. 2008, A\&A, 486, 637

Mathys, G. 2007, Highlights of Astronomy, 14, 285

McLaughlin, D. B. 1924, ApJ, 60, 22

de Medeiros, J. R., \& Mayor, M. 1999, A\&AS, 139, 433

Mermilliod, J.-C., Grenon, M., \& Mayor, M. 2008, A\&A, 491, 951

Moffett, T. J., \& Barnes III., T. G. 1979, PASP, 91, 180

Nicolet, B. 1978, A\&AS, 34, 1

Odenkirchen, M., Soubiran, C., \& Colin, J. 1998, New Astron., 3, 583

Pavlenko, Ya. V., \& Magazzú, A. 1996, A\&A, 311, 961

Paxton, W. et al. 2010, Modules for Experiments in Stellar Astrophysics (MESA) http://mesa. sourceforge.ne

Pietrinferni, A., Cassisi, S., Salaris, M., \& Castelli, F. 2004, ApJ, 612, 168

Piskunov, N. E., \& Rice, J. B. 1993, PASP, 105, 1415

Piskunov, N. E., \& Wehlau, W. H. 1994, A\&A, 289, 868

Pizzolato, N., Maggio, A., Micela, G., Sciortino, S., \& Ventura, P. 2003, A\&A, 397, 147

Plachinda, S. I. 2005, Astrophys., 48, 1, 9

Press, W. H., Teukolsky, S. A., Vetterling, W. T., \& Flannery, B. P. 2002, Numerical Recipes, 2nd edn (Cambridge: Cambridge University Press)

Reegen, P. 2004, in Second Eddington Workshop: Stellar structure and habitable planet finding, ed. F. Favata, S. Aigrain, \& A. Wilson, ESA SP, 583, 389 Reegen, P. 2007, A\&A, 467, 1353

Reegen, P., Kallinger, T., Frast, D., et al. 2006, MNRAS, 367, 1414

Reiners, A. 2006, A\&A, 446, 267

Rice, J. B., \& Strassmeier, K. G. 2000, A\&AS, 147, 151

Richichi, A., Percheron, I., \& Khristoforova, M. 2005, A\&A, 431, 773

Roberts, D. H., Lehar, J., \& Dreher, J. W. 1987, AJ, 93, 968

Rossiter, R. A. 1924, ApJ, 60, 15

Rowe, J. F. 2006, ApJ, 646, 1241

Saar, S. H., \& Donahue, R. A. 1997, ApJ, 485, 319

Saar, S. H., Huovelin, J., Osten, R. A., \& Shcherbakov, A. G. 1997, A\&A, 326, 741

Scargle, J. D. 1982, ApJ, 263, 835

Scelsi, L., Maggio, A., Peres, G., \& Gondoin, P. 2004, A\&A, 413, 643

Schrijver, C. J., \& Zwaan, C. 2000, Solar and stellar magnetic aczivity (Cambridge U.K.: Cambridge University Press)

Schüssler, M., \& Solanki, S. K. 1992, A\&A, 264, L13

Simon, T. 1984, ApJ, 279, 738

Simon, T. 1986, AJ, 91, 1233

Simon, T., \& Drake, S. A. 1989, ApJ, 346, 303

Skumanich, A. 1972, ApJ, 171, 565

Stellingwerf, R. F. 1978, ApJ, 224, 953

Strassmeier, K. G. 2009, A\&ARv, 17, 251

Strassmeier, K. G., \& Hall, D. S. 1988, ApJS, 67, 439

Strassmeier, K. G., \& Oláh, K. 2004, in Second Eddington Workshop: Stellar structure and habitable planet finding, ed. F. Favata, S. Aigrain, \& A. Wilson, ESA SP, 583, 149

Strassmeier, K. G., Fekel, F. C., Bopp, B. W., Dempsey, R. C., \& Henry, G. W. 1990, ApJS, 72, 191

Strassmeier, K. G., Washüttl, A., \& Rice, J. B. 1994, IBVS, 3994

Strassmeier, K. G., Bartus, J., Cutispoto, G., \& Rodonó, M. 1997a, A\&AS, 125, 11

Strassmeier, K. G., Boyd, L. J., Epand, D. H., \& Granzer, T. 1997b, PASP, 109, 697

Strassmeier, K. G., Serkowitsch, E., \& Granzer, T. 1999, A\&AS, 140, 29

Strassmeier, K. G., Reegen, P., \& Granzer, T. 2001, AN, 322, 325

Strassmeier, K. G., Granzer, T., Weber, M., et al. 2004, AN, 325, 527

Strassmeier, K. G., Weber, M., Granzer, T., \& Dall, T. H. 2010, AN, 331, 368

van Leeuwen, F. 2007, A\&A, 474, 653

Vogt, S. S., Penrod, G. D., \& Hatzes, A. P. 1987, ApJ, 321, 496

Walker, G., Matthews, J., Kuschnig, R., et al. 2003, PASP, 115, 1023

Wallerstein, G., Böhm-Vitense, E., Vanture, A. D., \& Gonzalez, G. 1994, AJ, 107,2211

Weber, M., Granzer, T., Strassmeier, K. G., \& Woche, M. 2008, Proc. SPIE, 7019, 70190L

Wittkowski, M., Schöller, M., Hubrig, S., Posselt, B., \& von der Lühe, O. 2002, AN, 323,241 